\title{
Hausdorff quasi-distances, periodic and fixed points for Nadler type set-valued contractions in quasi-gauge spaces
}

Kazimierz Włodarczyk

\section{"Correspondence:}

wlkzxa@math.uni.lodz.pl

Department of Nonlinear Analysis,

Faculty of Mathematics and

Computer Science, University of

Łódź, Banacha 22, Łódź, 90-238,

Poland

\section{空 Springer}

\begin{abstract}
In a quasi-gauge space $(X, \mathcal{P})$ with quasi-gauge $\mathcal{P}$, using the left (right) $\mathcal{J}$-families of generalized quasi-pseudodistances on $X$ ( $\mathcal{J}$-families on $X$ generalize quasi-gauge $\mathcal{P}$ ), the left (right) quasi-distances $\mathcal{D}_{\eta}^{L-\mathcal{J}}\left(\mathcal{D}_{\eta}^{R-\mathcal{J}}\right)$ of Hausdorff type on $2^{X}$ are defined, $\eta \in\{1,2,3\}$, the three kinds of left (right) set-valued contractions of Nadler type are constructed, and, for such contractions, the left (right) $\mathcal{P}$-convergence of dynamic processes starting at each point $w^{0} \in X$ is studied and the existence and localization of periodic and fixed point results are proved. As implications, two kinds of left (right) single-valued contractions of Banach type are defined, and, for such contractions, the left (right) $\mathcal{P}$-convergence of Picard iterations starting at each point $w^{0} \in X$ is studied, and existence, localization, periodic point, fixed point and uniqueness results are established. Appropriate tools and ideas of studying based on $\mathcal{J}$-families and also presented examples showed that the results: are new in quasi-gauge, topological, gauge, quasi-uniform and quasi-metric spaces; are new even in uniform and metric spaces; do not require completeness and Hausdorff properties of the spaces $(X, \mathcal{P})$, continuity of contractions, closedness of values of set-valued contractions and properties $\mathcal{D}_{\eta}^{L-\mathcal{J}}(U, V)=\mathcal{D}_{\eta}^{L-\mathcal{J}}(V, U)\left(\mathcal{D}_{\eta}^{R-\mathcal{J}}(U, V)=\mathcal{D}_{\eta}^{R-\mathcal{J}}(V, U)\right)$ and $\mathcal{D}_{\eta}^{L-\mathcal{J}}(U, U)=0$ $\left(\mathcal{D}_{\eta}^{R-\mathcal{J}}(U, U)=0\right), \eta \in\{1,2,3\}, U, V \in 2^{X}$; provide information concerning localizations of periodic and fixed points; and substantially generalize the well-known theorems of Nadler and Banach types.

MSC: 54A05; 54C60; 47H09; 37C25; 54H20; 54H25; 54E15

Keywords: Hausdorff distance; Nadler contraction; Banach contraction; periodic point; fixed point; localization; quasi-gauge space; generalized quasi-pseudodistance; dynamic system; convergence of dynamic process
\end{abstract}

\section{Introduction}

There are in the literature many different versions of the well-known theorems due to Banach [1] and Nadler [2] concerning fixed points for single-valued and set-valued dynamic systems, respectively, in complete metric spaces. Especially, their analogues in more general spaces and concerning nontrivial problems and more complicated situations are important, fascinating and challenging ( $c f$. [3-59]).

Recall that a set-valued dynamic system is defined as a pair $(X, T)$, where $X$ is a certain space and $T$ is a set-valued map $T: X \rightarrow 2^{X}$; here $2^{X}$ denotes the family of all nonempty subsets of a space $X$. In particular, a set-valued dynamic system includes the single-valued

@2014 Włodarczyk; licensee Springer. This is an Open Access article distributed under the terms of the Creative Commons Attribution License (http://creativecommons.org/licenses/by/2.0), which permits unrestricted use, distribution, and reproduction in any medium, provided the original work is properly cited. 
dynamic system $(X, T)$ where $T$ is a single-valued map $T: X \rightarrow X$, i.e., $\forall_{x \in X}\{T(x) \in X\}$. For $m \in\{0\} \cup \mathbb{N}$, define $T^{[m]}=T \circ T \circ \cdots \circ T$ ( $m$-times) and $T^{[0]}=I_{X}$ (an identity map on $X$ ).

Let $(X, T)$ be a set-valued dynamic system. By $\operatorname{Fix}(T)$ and $\operatorname{Per}(T)$ we denote the sets of all fixed points and periodic points of $T$, respectively, i.e., $\operatorname{Fix}(T)=\{w \in X: w \in T(w)\}$ and $\operatorname{Per}(T)=\left\{w \in X: w \in T^{[s]}(w)\right.$ for some $\left.s \in \mathbb{N}\right\}$. A dynamic process or a trajectory starting at $w_{0} \in X$ or a motion of the system $(X, T)$ at $w^{0}$ is a sequence $\left(w^{m}: m \in\{0\} \cup \mathbb{N}\right)$ defined by $w^{m} \in T\left(w^{m-1}\right)$ for $m \in \mathbb{N}$ (see Aubin and Siegel [5], Aubin and Ekeland [60], Aubin and Frankowska [4] and Yuan [58]).

Let $(X, T)$ be a single-valued dynamic system. For each $w^{0} \in X$, a sequence $\left(w^{m}: m \in\right.$ $\{0\} \cup \mathbb{N}$ ) such that $\forall_{m \in\{0\} \cup \mathbb{N}}\left\{w^{m}=T^{[m]}\left(w^{0}\right)\right\}$ is called a Picard iteration starting at $w^{0}$ of the system $(X, T)$.

The notion of Banach's contraction belongs to the most fundamental mathematical ideas and a classic result of Banach, from 1922, is the milestone in the history of fixed point theory and its applications.

Theorem 1.1 (Banach [1]) Let $(X, d)$ be a complete metric space. Assume that the singlevalued dynamic system $(X, T)$ is $(d, \lambda)$-contraction, i.e.,

$$
\exists_{\lambda \in[0 ; 1)} \forall_{x, y \in X}\{d(T(x), T(y)) \leq \lambda d(x, y)\} .
$$

Then $T$ has a unique fixed point $w$ in $X$ (i.e., $T(w)=w$ and $\operatorname{Fix}(T)=\{w\})$ and, for each $w^{0} \in X$, the sequence $\left(w^{m}=T^{[m]}\left(w_{0}\right): m \in\{0\} \cup \mathbb{N}\right)$ satisfies $\lim _{m \rightarrow \infty} d\left(w, w^{m}\right)=0$.

Recall that the Hausdorff metric $H^{d}$ on the class of all nonempty closed and bounded subsets $C B(X)$ of the metric space $(X, d)$ is defined as

$$
\forall U, V \in C B(X)\left\{H^{d}(U, V)=\max \left\{\sup _{u \in U} d(u, V), \sup _{v \in V} d(v, U)\right\}\right\}
$$

where $\forall_{u \in X} \forall_{V \in C B(X)}\left\{d(u, V)=\inf _{v \in V} d(u, v)\right\}$.

In a slightly different direction is the following elegant result of Nadler on set-valued dynamic systems.

Theorem 1.2 (Nadler [2, Theorem 5]) Let $(X, d)$ be a complete metric space. Assume that the set-valued dynamic system $(X, T)$ satisfying $T: X \rightarrow C B(X)$ is $\left(H^{d}, \lambda\right)$-contraction, i.e.,

$$
\exists_{\lambda \in[0 ; 1)} \forall_{x, y \in X}\left\{H^{d}(T(x), T(y)) \leq \lambda d(x, y)\right\} .
$$

Then $\operatorname{Fix}(T) \neq \varnothing$ (i.e., there exists $w \in X$ such that $w \in T(w))$.

Remark 1.1 Clearly, $(X, d)$ and $\left(C B(X), H^{d}\right)$, as metrics, are Hausdorff spaces, and the completeness of $(X, d)$ implies the completeness of $\left(C B(X), H^{d}\right)$. Observe that in the proofs of Theorems 1.1 and 1.2 the following play an important role: (a) the continuity of $d$ and $H^{d}$; (b) the completeness and the separability of the spaces $(X, d)$ and $\left(C B(X), H^{d}\right)$; (c) the continuity of maps $T:(X, d) \rightarrow(X, d)$ and $T:(X, d) \rightarrow\left(C B(X), H^{d}\right)$ satisfying conditions (1.1) and (1.3), respectively; (d) in Theorem 1.2 the assumption that for each $x \in X, T(x)$ is closed in $X$; (e) the properties $H^{d}(U, V)=H^{d}(V, U)$ and $H^{d}(U, U)=0, U, V \in C B(X)$. 
By analyzing Theorems 1.1 and 1.2, one may build many examples without properties (a)-(e) and such that the assertions are obtainable and remain valid. These remarks suggest that more subtle investigations and modifications of structures on $X$, and the concept of distance of Hausdorff defined by (1.2), and the concepts of contractions of Banach and Nadler defined by (1.1) and (1.3) respectively are necessary. The aim of this paper is to provide new modifications of Theorems 1.1 and 1.2 removing the assumptions (a)-(e) mentioned in Remark 1.1 and leaving the assertions such as in Theorems 1.1 and 1.2, even in more general forms.

More precisely, let $X$ be a nonempty set, let the family $\mathcal{P}=\left\{p_{\alpha}: \alpha \in \mathcal{A}\right\}$ of quasipseudometrics $p_{\alpha}: X \times X \rightarrow[0, \infty), \alpha \in \mathcal{A}$, be a quasi-gauge on $X$ such that $(X, \mathcal{P})$ is a quasi-gauge space (in the sense of Dugundji [61] and Reilly [62]), and let the family $\mathcal{J}=\left\{J_{\alpha}: \alpha \in \mathcal{A}\right\}$ of generalized quasi-pseudodistances $J_{\alpha}: X \times X \rightarrow[0, \infty), \alpha \in \mathcal{A}$, be a left (right) $\mathcal{J}$-family on $X$ ( $\mathcal{J}$-families on $X$ generalize quasi-gauge $\mathcal{P}$ on $X)$. Then, in $(X, \mathcal{P})$, using the left (right) $\mathcal{J}$-families on $X$, the left (right) quasi-distances $\mathcal{D}_{\eta}^{L-\mathcal{J}}\left(\mathcal{D}_{\eta}^{R-\mathcal{J}}\right)$, $\eta \in\{1,2,3\}$, of Hausdorff type on $2^{X}$ are defined, the three kinds of left (right) set-valued contractions of Nadler type are constructed, and, for such contractions, the left (right) $\mathcal{P}$-convergence of dynamic processes starting at each point $w^{0} \in X$ is studied and the existence and localization of periodic and fixed point results are proved. As implications, two kinds of left (right) single-valued contractions of Banach type are defined, the left (right) $\mathcal{P}$-convergence of Picard iterations starting at each point $w^{0} \in X$ is studied, and existence, localization, periodic point, fixed point and uniqueness results for such contractions are established.

The left (right) set-valued and single-valued contractions are studied here on $X$, on $\left\{w^{0}\right\} \cup B^{L-\mathcal{J}}\left(w^{0}, r\right)$ and on $\left\{w^{0}\right\} \cup B^{R-\mathcal{J}}\left(w^{0}, r\right)$, where $B^{L-\mathcal{J}}\left(w^{0}, r\right)\left(B^{R-\mathcal{J}}\left(w^{0}, r\right)\right)$ are left (right) $\mathcal{J}$-balls centered in $w^{0} \in X$ of radius $r=\left\{r_{\alpha}\right\}_{\alpha \in \mathcal{A}} \in(0 ; \infty)^{\mathcal{A}}$.

Moreover, in our investigations, we assume additionally that these left (right) contractions are left (right) $\mathcal{J}$-admissible or left (right) partially $\mathcal{J}$-admissible. Also, the cases when these left (right) contractions are left (right) $\mathcal{P}$-quasi-closed maps are described.

Appropriate tools and ideas of studying based on asymmetric structures determined by $\mathcal{J}$-families and also presented examples showed that the results: are new in quasi-gauge, topological, gauge, quasi-uniform and quasi-metric spaces; are new even in uniform and metric spaces; do not require completeness and Hausdorff properties of the spaces $(X, \mathcal{P})$, continuity of contractions, closedness of values of set-valued contractions and properties $\mathcal{D}_{\eta}^{L-\mathcal{J}}(U, V)=\mathcal{D}_{\eta}^{L-\mathcal{J}}(V, U)\left(\mathcal{D}_{\eta}^{R-\mathcal{J}}(U, V)=\mathcal{D}_{\eta}^{R-\mathcal{J}}(V, U)\right)$ and $\mathcal{D}_{\eta}^{L-\mathcal{J}}(U, U)=0$ $\left(\mathcal{D}_{\eta}^{R-\mathcal{J}}(U, U)=0\right), \eta \in\{1,2,3\}, U, V \in 2^{X}$; provide information concerning localizations of periodic and fixed points; and substantially generalize the well-known theorems of Nadler and Banach types.

\section{Quasi-gauge spaces}

Before proceeding further, let us record the following.

Definition 2.1 Let $X$ be a nonempty set.

(A) A quasi-pseudometric on $X$ is a map $p: X \times X \rightarrow[0, \infty)$ such that:

(a) $\forall_{u \in X}\{p(u, u)=0\}$; and (b) $\forall_{u, v, w \in X}\{p(u, w) \leq p(u, v)+p(v, w)\}$. For given quasi-pseudometric $p$ on $X$, a pair $(X, p)$ is called quasi-pseudometric space. 
A quasi-pseudometric space $(X, p)$ is called Hausdorff if

$$
\forall_{u, v \in X}\{u \neq v \Rightarrow p(u, v)>0 \vee p(v, u)>0\}
$$

(B) Each family $\mathcal{P}=\left\{p_{\alpha}: \alpha \in \mathcal{A}\right\}$ of quasi-pseudometrics $p_{\alpha}: X \times X \rightarrow[0, \infty), \alpha \in \mathcal{A}$, is called a quasi-gauge on $X$.

(C) Let the family $\mathcal{P}=\left\{p_{\alpha}: \alpha \in \mathcal{A}\right\}$ be a quasi-gauge on $X$. The topology $\mathcal{T}(\mathcal{P})$ having as a subbase the family $\mathcal{B}(\mathcal{P})=\left\{B\left(u, \varepsilon_{\alpha}\right): u \in X, \varepsilon_{\alpha}>0, \alpha \in \mathcal{A}\right\}$ of all balls $B\left(u, \varepsilon_{\alpha}\right)=\left\{v \in X: p_{\alpha}(u, v)<\varepsilon_{\alpha}\right\}, u \in X, \varepsilon_{\alpha}>0, \alpha \in \mathcal{A}$, is called the topology induced by $\mathcal{P}$ on $X$.

(D) (Dugundji [61], Reilly [34, 62]) A topological space $(X, \mathcal{T})$ such that there is a quasi-gauge $\mathcal{P}$ on $X$ with $\mathcal{T}=\mathcal{T}(\mathcal{P})$ is called a quasi-gauge space and is denoted by $(X, \mathcal{P})$.

(E) A quasi-gauge space $(X, \mathcal{P})$ is called Hausdorff if the quasi-gauge $\mathcal{P}$ has the property:

$$
\forall_{u, v \in X}\left\{u \neq v \Rightarrow \exists_{\alpha \in \mathcal{A}}\left\{p_{\alpha}(u, v)>0 \vee p_{\alpha}(v, u)>0\right\}\right\}
$$

(F) Let the family $\mathcal{P}=\left\{p_{\alpha}: \alpha \in \mathcal{A}\right\}$ be a quasi-gauge on $X$, and let $(X, \mathcal{P})$ be a quasi-gauge space. If $\mathcal{P}^{\prime}=\left\{p_{\alpha}^{\prime}: \alpha \in \mathcal{A}\right\}$, where $\forall_{\alpha \in \mathcal{A}} \forall_{u, v \in X}\left\{p_{\alpha}^{\prime}(u, v)=p_{\alpha}(v, u)\right\}$, then $\left(X, \mathcal{P}^{\prime}\right)$ is a quasi-gauge space and $\left(X, \mathcal{P}^{\prime}\right)$ is called the conjugate of $(X, \mathcal{P})$.

Remark 2.1 Each quasi-uniform space and each topological space is a quasi-gauge space (Reilly [62, Theorems 4.2 and 2.6]). The quasi-gauge spaces are the greatest general spaces with asymmetric structures.

\section{Left (right) $\mathcal{J}$-families}

Historically, the first work on the distances in metric spaces $(X, d)$ was done by Tataru [45]. Next, the concepts of $w$-distances, $\tau$-functions and $\tau$-distances in these spaces, which generalize Tataru distances and metrics $d$, were introduced by Kada et al. [23], Lin and Du [27] and Suzuki [37], respectively. Distances in uniform spaces had first been formulated by Vályi [46]. From rich literature it follows that the above distances provide useful and powerful tools for investigating problems of fixed point theory. Using these ideas, more general and various distances have been demonstrated in [47-57].

For a different purpose, in quasi-gauge spaces $(X, \mathcal{P})$ with quasi-gauges $\mathcal{P}=\left\{p_{\alpha}: \alpha \in\right.$ $\mathcal{A}$ \} on $X$, we recall the left (right) $\mathcal{J}$-families of generalized quasi-pseudodistances on $X$ (left (right) $\mathcal{J}$-families generalize quasi-gauges $\mathcal{P}$ ).

Definition 3.1 ([57, Section 3]) Let $(X, \mathcal{P})$ be a quasi-gauge space.

(A) The family $\mathcal{J}=\left\{J_{\alpha}: \alpha \in \mathcal{A}\right\}$ of maps $J_{\alpha}: X \times X \rightarrow[0, \infty), \alpha \in \mathcal{A}$, is said to be a left (right) $\mathcal{J}$-family of generalized quasi-pseudodistances on $X$ (left (right) $\mathcal{J}$-family on $X$, for short) if the following two conditions hold:

(J) $\forall_{\alpha \in \mathcal{A}} \forall_{u, v, w \in X}\left\{J_{\alpha}(u, w) \leq J_{\alpha}(u, v)+J_{\alpha}(v, w)\right\}$; and

$(\mathcal{J} 2)$ for any sequences $\left(u_{m}: m \in \mathbb{N}\right)$ and $\left(v_{m}: m \in \mathbb{N}\right)$ in $X$ satisfying

$$
\forall_{\alpha \in \mathcal{A}}\left\{\lim _{m \rightarrow \infty} \sup _{n>m} J_{\alpha}\left(u_{m}, u_{n}\right)=0\right\}
$$




$$
\left(\forall_{\alpha \in \mathcal{A}}\left\{\lim _{m \rightarrow \infty} \sup _{n>m} J_{\alpha}\left(u_{n}, u_{m}\right)=0\right\}\right)
$$

and

$$
\begin{aligned}
& \forall_{\alpha \in \mathcal{A}}\left\{\lim _{m \rightarrow \infty} J_{\alpha}\left(v_{m}, u_{m}\right)=0\right\} \\
& \left(\forall_{\alpha \in \mathcal{A}}\left\{\lim _{m \rightarrow \infty} J_{\alpha}\left(u_{m}, v_{m}\right)=0\right\}\right),
\end{aligned}
$$

the following holds:

$$
\begin{aligned}
& \forall_{\alpha \in \mathcal{A}}\left\{\lim _{m \rightarrow \infty} p_{\alpha}\left(v_{m}, u_{m}\right)=0\right\} \\
& \left(\forall_{\alpha \in \mathcal{A}}\left\{\lim _{m \rightarrow \infty} p_{\alpha}\left(u_{m}, v_{m}\right)=0\right\}\right) .
\end{aligned}
$$

(B) Define

$$
\begin{aligned}
& \mathbb{J}_{(X, \mathcal{P})}^{L}=\left\{\mathcal{J}: \mathcal{J}=\left\{J_{\alpha}: \alpha \in \mathcal{A}\right\} \text { is a left } \mathcal{J} \text {-family on } X\right\}, \\
& \mathbb{J}_{(X, \mathcal{P})}^{R}=\left\{\mathcal{J}: \mathcal{J}=\left\{J_{\alpha}: \alpha \in \mathcal{A}\right\} \text { is a right } \mathcal{J} \text {-family on } X\right\}
\end{aligned}
$$

In the following remark, we list some basic properties of left (right) $\mathcal{J}$-families in $(X, \mathcal{P})$.

Remark 3.1 Let $(X, \mathcal{P})$ be a quasi-gauge space. The following hold:

(a) $\mathcal{P} \in \mathbb{J}_{(X, \mathcal{P})}^{L} \cap \mathbb{J}_{(X, \mathcal{P})}^{R}$.

(b) Let $\mathcal{J} \in \mathbb{J}_{(X, \mathcal{P})}^{L}$ or $\mathcal{J} \in \mathbb{J}_{(X, \mathcal{P})}^{R}$. If $\forall_{\alpha \in \mathcal{A}} \forall_{u \in X}\left\{J_{\alpha}(u, u)=0\right\}$, then for each $\alpha \in \mathcal{A}$, $J_{\alpha}$ is quasi-pseudometric.

(c) There are examples of $\mathcal{J} \in \mathbb{J}_{(X, \mathcal{P})}^{L}$ and $\mathcal{J} \in \mathbb{J}_{(X, \mathcal{P})}^{R}$ such that the maps $J_{\alpha}, \alpha \in \mathcal{A}$, are not quasi-pseudometrics. Indeed, in Example 4.1 below, if $u \notin E$, then $\forall_{\alpha \in \mathcal{A}}\left\{J_{\alpha}(u, u)=c_{\alpha}>0\right\}$.

(d) ([57, Proposition 3.1]) If $(X, \mathcal{P})$ is a Hausdorff quasi-gauge space and $\mathcal{J} \in \mathbb{J}_{(X, \mathcal{P})}^{L}$ or $\mathcal{J} \in \mathbb{J}_{(X, \mathcal{P})}^{R}$, then $\forall_{u, v \in X}\left\{u \neq v \Rightarrow \exists_{\alpha \in \mathcal{A}}\left\{J_{\alpha}(u, v)>0 \vee J_{\alpha}(v, u)>0\right\}\right\}$.

\section{Left (right) $\mathcal{J}$-balls}

In this section we define and characterize the left (right) $\mathcal{J}$-balls in $(X, \mathcal{P})$.

Definition 4.1 Let $(X, \mathcal{P})$ be a quasi-gauge space, and let the family $\mathcal{J}=\left\{J_{\alpha}: \alpha \in \mathcal{A}\right\}$ of maps $J_{\alpha}: X^{2} \rightarrow[0 ; \infty), \alpha \in \mathcal{A}$, be a left (right) $\mathcal{J}$-family on $X$. We define the left (right) $\mathcal{J}$-ball centered in $w^{0} \in X$ of radius $r=\left\{r_{\alpha}\right\}_{\alpha \in \mathcal{A}} \in(0 ; \infty)^{\mathcal{A}}$ by

$$
\begin{aligned}
& B^{L-\mathcal{J}}\left(w^{0}, r\right)=\left\{x \in X: \forall_{\alpha \in \mathcal{A}}\left\{J_{\alpha}\left(w^{0}, x\right) \leq r_{\alpha}\right\}\right\} \\
& \left(B^{R-\mathcal{J}}\left(w^{0}, r\right)=\left\{x \in X: \forall_{\alpha \in \mathcal{A}}\left\{J_{\alpha}\left(x, w^{0}\right) \leq r_{\alpha}\right\}\right\}\right) .
\end{aligned}
$$

Remark 4.1 Notice, however, that there exist a quasi-gauge space $(X, \mathcal{P})$, a left (right) $\mathcal{J}$-family on $X, w^{0} \in X$ and $r=\left\{r_{\alpha}\right\}_{\alpha \in \mathcal{A}} \in(0 ; \infty)^{\mathcal{A}}$ such that $w^{0} \notin B^{L-\mathcal{J}}\left(w^{0}, r\right)\left(w^{0} \notin\right.$ $\left.B^{R-\mathcal{J}}\left(w^{0}, r\right)\right)$. This follows from Example 4.1 below. 
Example 4.1 Let $X$ contain at least two different points, let the family $\mathcal{P}=\left\{p_{\alpha}: \alpha \in \mathcal{A}\right\}$ of quasi-pseudometrics $p_{\alpha}: X \times X \rightarrow[0, \infty), \alpha \in \mathcal{A}$, be a quasi-gauge on $X$, and let $(X, \mathcal{P})$ be a quasi-gauge space.

Let the set $E \subset X$ containing at least two different points be arbitrary and fixed, and let $\left\{c_{\alpha}\right\}_{\alpha \in \mathcal{A}} \in(0 ; \infty)^{\mathcal{A}}$ satisfy $\forall_{\alpha \in \mathcal{A}}\left\{\delta_{\alpha}(E)<c_{\alpha}\right\}$, where $\forall_{\alpha \in \mathcal{A}}\left\{\delta_{\alpha}(E)=\sup \left\{p_{\alpha}(x, y): x, y \in E\right\}\right\}$.

Let the family $\mathcal{J}=\left\{J_{\alpha}: \alpha \in \mathcal{A}\right\}, J_{\alpha}: X \times X \rightarrow[0, \infty), \alpha \in \mathcal{A}$, be defined by the formula:

$$
J_{\alpha}(x, y)=\left\{\begin{array}{ll}
p_{\alpha}(x, y) & \text { if } E \cap\{x, y\}=\{x, y\}, \\
c_{\alpha} & \text { if } E \cap\{x, y\} \neq\{x, y\},
\end{array} \quad x, y \in X\right.
$$

Then $\mathcal{J} \in \mathbb{J}_{(X, \mathcal{P})}^{L} \cap \mathbb{J}_{(X, \mathcal{P})}^{R}$.

Indeed, we see that condition $(\mathcal{J} 1)$ does not hold only if there exist some $\alpha \in \mathcal{A}$ and $u, v, w \in X$ such that $J_{\alpha}(u, w)=c_{\alpha}, J_{\alpha}(u, v)=p_{\alpha}(u, v), J_{\alpha}(v, w)=p_{\alpha}(v, w)$ and $p_{\alpha}(u, v)+$ $p_{\alpha}(v, w)<c_{\alpha}$. However, then we conclude that there exists $z \in\{u, w\}$ such that $z \notin E$ and $u, v, w \in E$, which is impossible. Therefore, $\forall_{\alpha \in \mathcal{A}} \forall_{u, v, w \in X}\left\{J_{\alpha}(u, w) \leq J_{\alpha}(u, v)+J_{\alpha}(v, w)\right\}$, i.e., condition $(\mathcal{J} 1)$ holds.

Now suppose that the sequences $\left\{u_{m}\right\}$ and $\left\{v_{m}\right\}$ in $X$ satisfy (3.1) and (3.3). Then, in particular, (3.3) yields

$$
\forall_{\alpha \in \mathcal{A}} \forall_{0<\varepsilon<c_{\alpha}} \exists_{m_{0}=m_{0}(\alpha) \in \mathbb{N}} \forall_{m \geq m_{0}}\left\{J_{\alpha}\left(v_{m}, u_{m}\right)<\varepsilon\right\} .
$$

By (4.2) and (4.1), denoting $m^{\prime}=\min \left\{m_{0}(\alpha): \alpha \in \mathcal{A}\right\}$, we conclude that

$$
\forall_{m \geq m^{\prime}}\left\{E \cap\left\{v_{m}, u_{m}\right\}=\left\{v_{m}, u_{m}\right\}\right\} .
$$

From (4.3), the definition of $\mathcal{J}$ and (4.2), we get

$$
\forall_{\alpha \in \mathcal{A}} \forall_{0<\varepsilon<c_{\alpha}} \exists_{m^{\prime} \in \mathbb{N}} \forall_{m \geq m^{\prime}}\left\{p_{\alpha}\left(v_{m}, u_{m}\right)=J_{\alpha}\left(v_{m}, u_{m}\right)<\varepsilon\right\} .
$$

The result is that the sequences $\left\{u_{m}\right\}$ and $\left\{v_{m}\right\}$ satisfy (3.5). Therefore, $\mathcal{J}$ is a left $\mathcal{J}$-family. Analogously, we prove that if $\left\{u_{m}\right\}$ and $\left\{v_{m}\right\}$ in $X$ satisfy (3.2) and (3.4), then also (3.6) holds, therefore $\mathcal{J}$ is a right $\mathcal{J}$-family.

\section{Left (right) $\mathcal{J}$-convergences and left (right) $\mathcal{J}$-sequential completeness}

Now, using left (right) $\mathcal{J}$-families, we define the following natural concept of left (right) $\mathcal{J}$-completeness in $(X, \mathcal{P})$.

Definition 5.1 Let $(X, \mathcal{P})$ be a quasi-gauge space, and let $\mathcal{J}=\left\{J_{\alpha}: \alpha \in \mathcal{A}\right\}$ be a left (right) $\mathcal{J}$-family on $X$.

(A) We say that a sequence $\left(u_{m}: m \in \mathbb{N}\right)$ in $X$ is left (right) $\mathcal{J}$-Cauchy sequence in $X$ if

$$
\begin{aligned}
& \forall_{\alpha \in \mathcal{A}}\left\{\lim _{m \rightarrow \infty} \sup _{n>m} J_{\alpha}\left(u_{m}, u_{n}\right)=0\right\} \\
& \left(\forall_{\alpha \in \mathcal{A}}\left\{\lim _{m \rightarrow \infty} \sup _{n>m} J_{\alpha}\left(u_{n}, u_{m}\right)=0\right\}\right) .
\end{aligned}
$$


(B) Let $u \in X$ and let $\left(u_{m}: m \in \mathbb{N}\right)$ be a sequence in $X$. We say that $\left(u_{m}: m \in \mathbb{N}\right)$ is left (right) $\mathcal{J}$-convergent to $u$ if $\lim _{m \rightarrow \infty}^{L-\mathcal{J}} u_{m}=u\left(\lim _{m \rightarrow \infty}^{R-\mathcal{J}} u_{m}=u\right)$, where

$$
\begin{aligned}
& \lim _{m \rightarrow \infty}^{L-\mathcal{J}} u_{m}=u \Leftrightarrow \forall_{\alpha \in \mathcal{A}}\left\{\lim _{m \rightarrow \infty} J_{\alpha}\left(u, u_{m}\right)=0\right\} \\
& \left(\lim _{m \rightarrow \infty}^{R-\mathcal{J}} u_{m}=u \Leftrightarrow \forall_{\alpha \in \mathcal{A}}\left\{\lim _{m \rightarrow \infty} J_{\alpha}\left(u_{m}, u\right)=0\right\}\right) .
\end{aligned}
$$

(C) We say that a sequence $\left(u_{m}: m \in \mathbb{N}\right)$ in $X$ is left (right) $\mathcal{J}$-convergent in $X$ if $S_{\left(u_{m}: m \in \mathbb{N}\right)}^{L-\mathcal{J}} \neq \varnothing\left(S_{\left(u_{m}: m \in \mathbb{N}\right)}^{R-\mathcal{J}} \neq \varnothing\right)$, where

$$
\begin{aligned}
& S_{\left(u_{m}: m \in \mathbb{N}\right)}^{L-\mathcal{J}}=\left\{u \in X: \lim _{m \rightarrow \infty}^{L-\mathcal{J}} u_{m}=u\right\} \\
& \left(S_{\left(u_{m}: m \in \mathbb{N}\right)}^{R-\mathcal{J}}=\left\{u \in X: \lim _{m \rightarrow \infty}^{R-\mathcal{J}} u_{m}=u\right\}\right) .
\end{aligned}
$$

(D) If every left (right) $\mathcal{J}$-Cauchy sequence $\left(u_{m}: m \in \mathbb{N}\right)$ in $X$ is left (right)

$\mathcal{J}$-convergent in $X$ (i.e., $\left.S_{\left(u_{m}: m \in \mathbb{N}\right)}^{L-\mathcal{J}} \neq \varnothing\left(S_{\left(u_{m}: m \in \mathbb{N}\right)}^{R-\mathcal{J}} \neq \varnothing\right)\right)$, then $(X, \mathcal{P})$ is called a left (right) $\mathcal{J}$-sequentially complete quasi-gauge space.

Remark 5.1 Let $(X, \mathcal{P})$ be a quasi-gauge space.

(a) It is clear that if $\left(u_{m}: m \in \mathbb{N}\right)$ is left (right) $\mathcal{J}$-convergent in $X$, then

$$
S_{\left(u_{m}: m \in \mathbb{N}\right)}^{L-\mathcal{J}} \subset S_{\left(v_{m}: m \in \mathbb{N}\right)}^{L-\mathcal{J}} \quad\left(S_{\left(u_{m}: m \in \mathbb{N}\right)}^{R-\mathcal{J}} \subset S_{\left(v_{m}: m \in \mathbb{N}\right)}^{R-\mathcal{J}}\right)
$$

for each subsequence $\left(v_{m}: m \in \mathbb{N}\right)$ of $\left(u_{m}: m \in \mathbb{N}\right)$.

(b) There exist examples of quasi-gauge spaces $(X, \mathcal{P})$ and left (right) $\mathcal{J}$-families $\mathcal{J}$ on $X, \mathcal{J} \neq \mathcal{P}$, such that $(X, \mathcal{P})$ are left (right) $\mathcal{J}$-sequentially complete, but not left (right) $\mathcal{P}$-sequentially complete.

\section{Left (right) $\mathcal{J}$-closed sets}

Definition 6.1 Let $(X, \mathcal{P})$ be a quasi-gauge space, and let the family $\mathcal{J}=\left\{J_{\alpha}: \alpha \in \mathcal{A}\right\}$ of maps $J_{\alpha}: X^{2} \rightarrow[0 ; \infty), \alpha \in \mathcal{A}$, be a left (right) $\mathcal{J}$-family on $X$.

(A) We say that a set $Y \in 2^{X}$ is a left (right) $\mathcal{J}$-closed in $X$ if $Y=c l_{X}^{L-\mathcal{J}}(Y)$ $\left(Y=c l_{X}^{R-\mathcal{J}}(Y)\right)$, where $c l_{X}^{L-\mathcal{J}}(Y)\left(c l_{X}^{R-\mathcal{J}}(Y)\right)$, the left (right) $\mathcal{J}$-closure in $X$, denotes the set of all $x \in X$ for which there exists a sequence $\left(x_{m}: m \in \mathbb{N}\right)$ in $Y$ which left (right) $\mathcal{J}$-converges to $x$.

(B) Define $C l^{L-\mathcal{J}}(X)=\left\{Y \in 2^{X}: Y=c l_{X}^{L-\mathcal{J}}(Y)\right\}\left(C l^{R-\mathcal{J}}(X)=\left\{Y \in 2^{X}: Y=c l_{X}^{R-\mathcal{J}}(Y)\right\}\right)$; that is, $C l^{L-\mathcal{J}}(X)\left(C l^{R-\mathcal{J}}(X)\right)$ denotes the class of all nonempty left (right) $\mathcal{J}$-closed subsets of $X$.

Remark 6.1 If $(X, \mathcal{P})$ is a left (right) $\mathcal{J}$-sequentially complete quasi-gauge space and a set $Y \in C l^{L-\mathcal{J}}(X)\left(Y \in C l^{R-\mathcal{J}}(X)\right)$, then $(Y, \mathcal{P})$ is a left (right) $\mathcal{J}$-sequentially complete quasigauge space.

\section{Left (right) $\mathcal{J}$-admissible and left (right) partially $\mathcal{J}$-admissible set-valued maps}

The following terminologies will be much used in the sequel. 
Definition 7.1 Let $(X, \mathcal{P})$ be a quasi-gauge space, let the family $\mathcal{J}=\left\{J_{\alpha}: \alpha \in \mathcal{A}\right\}$ of maps $J_{\alpha}: X \times X \rightarrow[0, \infty), \alpha \in \mathcal{A}$, be a left (right) $\mathcal{J}$-family on $X$, and let $Y \in 2^{X}$.

(A) We say that a set-valued map $T: Y \rightarrow 2^{X}$ is left (right) $\mathcal{J}$-admissible in a point $w^{0} \in Y$ if for each sequence $\left(w^{m}: m \in\{0\} \cup \mathbb{N}\right)$ satisfying the properties $\forall_{m \in\{0\} \cup \mathbb{N}}\left\{w^{m+1} \in T\left(w^{m}\right) \cap Y \neq \varnothing\right\}$ and

$$
\forall_{\alpha \in \mathcal{A}}\left\{\lim _{m \rightarrow \infty} \sup _{n>m} J_{\alpha}\left(w^{m}, w^{n}\right)=0\right\} \quad\left(\forall_{\alpha \in \mathcal{A}}\left\{\lim _{m \rightarrow \infty} \sup _{n>m} J_{\alpha}\left(w^{n}, w^{m}\right)=0\right\}\right),
$$

there exists $w \in X$ such that

$$
\forall_{\alpha \in \mathcal{A}}\left\{\lim _{m \rightarrow \infty} J_{\alpha}\left(w, w^{m}\right)=0\right\} \quad\left(\forall_{\alpha \in \mathcal{A}}\left\{\lim _{m \rightarrow \infty} J_{\alpha}\left(w^{m}, w\right)=0\right\}\right) .
$$

We say that a set-valued map $T: Y \rightarrow 2^{X}$ is left (right) $\mathcal{J}$-admissible in $Y$ if $T: Y \rightarrow 2^{X}$ is left (right) $\mathcal{J}$-admissible in each point $w^{0} \in Y$.

(B) We say that a set-valued map $T: Y \rightarrow 2^{X}$ is left (right) partially $\mathcal{J}$-admissible in a point $w^{0} \in Y$ if for each sequence $\left(w^{m}: m \in\{0\} \cup \mathbb{N}\right)$ satisfying the properties $\forall_{m \in\{0\} \cup \mathbb{N}}\left\{w^{m+1} \in T\left(w^{m}\right) \cap Y \neq \varnothing\right\}$ and

$$
\forall_{\alpha \in \mathcal{A}}\left\{\lim _{m \rightarrow \infty} \sup _{n>m} J_{\alpha}\left(w^{m}, w^{n}\right)=0\right\} \quad\left(\forall_{\alpha \in \mathcal{A}}\left\{\lim _{m \rightarrow \infty} \sup _{n>m} J_{\alpha}\left(w^{n}, w^{m}\right)=0\right\}\right),
$$

there exists $w \in X$ such that

$$
\forall_{\alpha \in \mathcal{A}}\left\{\lim _{m \rightarrow \infty} J_{\alpha}\left(w, w^{m}\right)=\lim _{m \rightarrow \infty} J_{\alpha}\left(w^{m}, w\right)=0\right\} .
$$

We say that a set-valued map $T: Y \rightarrow 2^{X}$ is left (right) partially $\mathcal{J}$-admissible in $Y$ if $T: Y \rightarrow 2^{X}$ is left (right) partially $\mathcal{J}$-admissible in each point $w^{0} \in Y$.

Remark 7.1 Let $(X, \mathcal{P})$ be a quasi-gauge space, and let the family $\mathcal{J}=\left\{J_{\alpha}: \alpha \in \mathcal{A}\right\}$ of maps $J_{\alpha}: X \times X \rightarrow[0, \infty), \alpha \in \mathcal{A}$, be a left (right) $\mathcal{J}$-family on $X$.

(a) If $(X, \mathcal{P})$ is a left (right) $\mathcal{J}$-sequentially complete quasi-gauge space, then a set-valued dynamic system $(X, T), T: X \rightarrow 2^{X}$, is left (right) $\mathcal{J}$-admissible on $X$.

(b) If $(X, \mathcal{P})$ is a left (right) $\mathcal{J}$-sequentially complete quasi-gauge space and $\mathcal{J}$ is symmetric, i.e., $\forall_{\alpha \in \mathcal{A}} \forall_{u, v \in X}\left\{J_{\alpha}(u, v)=J_{\alpha}(v, u)\right\}$, then $(X, T)$ is left (right) partially $\mathcal{J}$-admissible on $X$.

(c) It is evident that each left (right) partially $\mathcal{J}$-admissible on $X$ a set-valued dynamic system $(X, T)$ is left (right) $\mathcal{J}$-admissible on $X$ but the converse not necessarily holds.

\section{Left (right) $\mathcal{P}$-quasi-closed maps}

We can define the following generalizations of continuity.

Definition 8.1 Let $(X, \mathcal{P})$ be a quasi-gauge space, let $(X, T)$ be a set-valued dynamic system, $T: X \rightarrow 2^{X}$, and let $s \in \mathbb{N}$. The map $T^{[s]}$ is said to be a left (right) $\mathcal{P}$-quasi-closed map on $X$ if for every sequence $\left(x_{m}: m \in \mathbb{N}\right)$ in $T^{[s]}(X)$, left (right) $\mathcal{P}$-converging in $X$ (thus 
$\left.S_{\left(x_{m}: m \in \mathbb{N}\right)}^{L-\mathcal{P}} \neq \varnothing\left(S_{\left(x_{m}: m \in \mathbb{N}\right)}^{R-\mathcal{P}} \neq \varnothing\right)\right)$ and having subsequences $\left(v_{m}: m \in \mathbb{N}\right)$ and $\left(u_{m}: m \in \mathbb{N}\right)$ satisfying

$$
\forall_{m \in \mathbb{N}}\left\{v_{m} \in T^{[s]}\left(u_{m}\right)\right\}
$$

the following property holds: there exists $x \in S_{\left(x_{m}: m \in \mathbb{N}\right)}^{L-\mathcal{P}}\left(x \in S_{\left(x_{m}: m \in \mathbb{N}\right)}^{R-\mathcal{P}}\right)$ such that $x \in T^{[s]}(x)$ $\left(x \in T^{[s]}(x)\right)$.

Definition 8.2 Let $(X, \mathcal{P})$ be a quasi-gauge space, let $Y$ be a nonempty subset of $X$, and let $T: Y \rightarrow 2^{X}$ be a set-valued map. The map $T$ is said to be a left (right) $\mathcal{P}$-quasi-closed map on $Y$ if for every sequence $\left(x_{m}: m \in \mathbb{N}\right)$ in $T(Y)$, left (right) $\mathcal{P}$-converging in $X$ (thus $\left.S_{\left(x_{m}: m \in \mathbb{N}\right)}^{L-\mathcal{P}} \neq \varnothing\left(S_{\left(x_{m}: m \in \mathbb{N}\right)}^{R-\mathcal{P}} \neq \varnothing\right)\right)$ and having subsequences $\left(v_{m}: m \in \mathbb{N}\right)$ and $\left(u_{m}: m \in \mathbb{N}\right)$ satisfying

$$
\forall_{m \in \mathbb{N}}\left\{v_{m} \in T\left(u_{m}\right)\right\}
$$

the following property holds: there exists $x \in S_{\left(x_{m}: m \in \mathbb{N}\right)}^{L-\mathcal{P}}\left(x \in S_{\left(x_{m}: m \in \mathbb{N}\right)}^{R-\mathcal{P}}\right)$ such that $x \in T(x)$ $(x \in T(x))$.

\section{Left (right) quasi-distances of Hausdorff type and three kinds of set-valued left (right) contractions of Nadler type}

In not necessarily Hausdorff quasi-gauge spaces, we define the left (right) Hausdorff quasidistances (Definition 9.1(A)) and the set-valued left (right) contractions of Nadler type (Definition 9.1(B)).

Definition 9.1 Let $(X, \mathcal{P})$ be a quasi-gauge space, let the family $\mathcal{J}=\left\{J_{\alpha}: \alpha \in \mathcal{A}\right\}$ of maps $J_{\alpha}: X^{2} \rightarrow[0 ; \infty), \alpha \in \mathcal{A}$, be a left (right) $\mathcal{J}$-family on $X$, let $\eta \in\{1,2,3\}$, and let

$$
\begin{gathered}
\forall_{\alpha \in \mathcal{A}} \forall_{u \in X} \forall_{V \in 2^{X}}\left\{J_{\alpha}(u, V)=\inf \left\{J_{\alpha}(u, z): z \in V\right\}\right. \\
\left.\wedge J_{\alpha}(V, u)=\inf \left\{J_{\alpha}(z, u): z \in V\right\}\right\} .
\end{gathered}
$$

(A) Define on $2^{X}$ the left (right) quasi-distance $\mathcal{D}_{\eta}^{L-\mathcal{J}}=\left\{D_{\eta ; \alpha}^{L-\mathcal{J}}, \alpha \in \mathcal{A}\right\}$ $\left(\mathcal{D}_{\eta}^{R-\mathcal{J}}=\left\{D_{\eta ; \alpha}^{R-\mathcal{J}}, \alpha \in \mathcal{A}\right\}\right)$ of Hausdorff type, where $D_{\eta ; \alpha}^{L-\mathcal{J}}: 2^{X} \times 2^{X} \rightarrow[0 ; \infty], \alpha \in \mathcal{A}$ $\left(D_{\eta ; \alpha}^{R-\mathcal{J}}: 2^{X} \times 2^{X} \rightarrow[0 ; \infty], \alpha \in \mathcal{A}\right)$ are defined as follows:

$$
\begin{aligned}
& \forall_{\alpha \in \mathcal{A}} \forall_{U, V \in 2^{X}}\left\{D_{1 ; \alpha}^{L-\mathcal{J}}(U, V)=\max \left\{\sup _{u \in U} J_{\alpha}(u, V), \sup _{v \in V} J_{\alpha}(U, v)\right\}\right\}, \\
& \forall_{\alpha \in \mathcal{A}} \forall_{U, V \in 2^{X}}\left\{D_{2 ; \alpha}^{L-\mathcal{J}}(U, V)=\max \left\{\sup _{u \in U} J_{\alpha}(u, V), \sup _{v \in V} J_{\alpha}(v, U)\right\}\right\} \text { and } \\
& \forall_{\alpha \in \mathcal{A}} \forall_{U, V \in 2^{X}}\left\{D_{3 ; \alpha}^{L-\mathcal{J}}(U, V)=\sup _{u \in U} J_{\alpha}(u, V)\right\} \text { if } \mathcal{J} \in \mathbb{J}_{(X, \mathcal{P})}^{L} ; \\
& \forall_{\alpha \in \mathcal{A}} \forall_{U, V \in 2^{X}}\left\{D_{1 ; \alpha}^{R-\mathcal{J}}(U, V)=\max \left\{\sup _{u \in U} J_{\alpha}(u, V), \sup _{v \in V} J_{\alpha}(U, v)\right\}\right\}, \\
& \forall_{\alpha \in \mathcal{A}} \forall_{U, V \in 2^{X}}\left\{D_{2 ; \alpha}^{R-\mathcal{J}}(U, V)=\max \left\{\sup _{u \in U} J_{\alpha}(u, V), \sup _{v \in V} J_{\alpha}(v, U)\right\}\right\} \text { and } \\
& \forall_{\alpha \in \mathcal{A}^{\forall}} \forall_{U, V \in 2^{X}}\left\{D_{3 ; \alpha}^{R-\mathcal{J}}(U, V)=\sup _{u \in U} J_{\alpha}(u, V)\right\} \text { if } \mathcal{J} \in \mathbb{J}_{(X, \mathcal{P})}^{R} .
\end{aligned}
$$

(B) Let $\lambda=\left\{\lambda_{\alpha}\right\}_{\alpha \in \mathcal{A}} \in[0 ; 1)^{\mathcal{A}}$ and let $Y \in 2^{X}$. We say that a set-valued map $T: Y \rightarrow 2^{X}$ is left (right) $\left(\mathcal{D}_{\eta}^{L-\mathcal{J}}, \lambda\right)$-contraction on $Y\left(\left(\mathcal{D}_{\eta}^{R-\mathcal{J}}, \lambda\right)\right.$-contraction on $\left.Y\right)$ if:

(B.1) $\forall_{\alpha \in \mathcal{A}} \forall_{x, y \in Y}\left\{D_{\eta ; \alpha}^{L-\mathcal{J}}(T(x), T(y)) \leq \lambda_{\alpha} J_{\alpha}(x, y)\right\}$ if $\mathcal{J} \in \mathbb{J}_{(X, \mathcal{P})}^{L}$;

(B.2) $\forall_{\alpha \in \mathcal{A}} \forall_{x, y \in Y}\left\{D_{\eta ; \alpha}^{R-\mathcal{J}}(T(x), T(y)) \leq \lambda_{\alpha} J_{\alpha}(x, y)\right\}$ if $\mathcal{J} \in \mathbb{J}_{(X, \mathcal{P})}^{R}$. 
Remark 9.1 Let $(X, \mathcal{P})$ be a quasi-gauge space, and let the family $\mathcal{J}=\left\{J_{\alpha}: \alpha \in \mathcal{A}\right\}$ of maps $J_{\alpha}: X^{2} \rightarrow[0 ; \infty), \alpha \in \mathcal{A}$, be a left (right) $\mathcal{J}$-family on $X$.

(a) Generally, $D_{\eta ; \alpha}^{L-\mathcal{J}}\left(D_{\eta ; \alpha}^{R-\mathcal{J}}\right)$ are not symmetric, i.e., $D_{\eta ; \alpha}^{L-\mathcal{J}}(U, V)=D_{\eta ; \alpha}^{L-\mathcal{J}}(V, U)$ $\left(D_{\eta ; \alpha}^{R-\mathcal{J}}(U, V)=D_{\eta ; \alpha}^{R-\mathcal{J}}(V, U)\right)$ not necessarily hold. Moreover, $\mathcal{D}_{\eta}^{L-\mathcal{J}}(U, U)=0$ $\left(\mathcal{D}_{\eta}^{R-\mathcal{J}}(U, U)=0\right)$ not necessarily hold; see Remarks 12.1 and 12.3 .

(b) Each $\left(\mathcal{D}_{\eta}^{L-\mathcal{J}}, \lambda\right)$-contraction on $Y\left(\left(\mathcal{D}_{\eta}^{R-\mathcal{J}}, \lambda\right)\right.$-contraction on $\left.Y\right), \eta \in\{1,2\}$, is $\left(\mathcal{D}_{3}^{L-\mathcal{J}}, \lambda\right)$-contraction on $Y\left(\left(\mathcal{D}_{3}^{R-\mathcal{J}}, \lambda\right)\right.$-contraction on $\left.Y\right)$ but the converse not necessarily holds.

\section{Convergence, existence, fixed point, periodic point and localization results for left (right) set-valued contractions of Nadler type}

We have the following theorem.

Theorem 10.1 Let $(X, \mathcal{P})$ be a quasi-gauge space, let the family $\mathcal{J}=\left\{J_{\alpha}: \alpha \in \mathcal{A}\right\}$ of maps $J_{\alpha}: X^{2} \rightarrow[0 ; \infty), \alpha \in \mathcal{A}$, be a left (right) $\mathcal{J}$-family on $X$, and suppose that $\eta \in\{1,2,3\}$. Assume, moreover, that $\lambda=\left\{\lambda_{\alpha}\right\}_{\alpha \in \mathcal{A}} \in[0 ; 1)^{\mathcal{A}}$ and a set-valued dynamic system $(X, T)$, $T: X \rightarrow 2^{X}$, satisfy the following:

(i) $T$ is $\left(\mathcal{D}_{\eta}^{L-\mathcal{J}}, \lambda\right)$-contraction on $X\left(T\right.$ is $\left(\mathcal{D}_{\eta}^{R-\mathcal{J}}, \lambda\right)$-contraction on $\left.X\right)$; and

(ii) For every $x \in X$ and for every $\gamma=\left\{\gamma_{\alpha}\right\}_{\alpha \in \mathcal{A}} \in(0 ; \infty)^{\mathcal{A}}$, there exists $y \in T(x)$ such that

$$
\begin{aligned}
& \forall_{\alpha \in \mathcal{A}}\left\{J_{\alpha}(x, y)<J_{\alpha}(x, T(x))+\gamma_{\alpha}\right\} \\
& \left(\forall_{\alpha \in \mathcal{A}}\left\{J_{\alpha}(y, x)<J_{\alpha}(T(x), x)+\gamma_{\alpha}\right\}\right) .
\end{aligned}
$$

(A) If $(X, T)$ is left (right) $\mathcal{J}$-admissible in a point $w^{0} \in X$, then there exist a dynamic process $\left(w^{m}: m \in\{0\} \cup \mathbb{N}\right)$ of the system $(X, T)$ starting at $w^{0}$, a point $w \in X$ and $r=\left\{r_{\alpha}\right\}_{\alpha \in \mathcal{A}} \in(0 ; \infty)^{\mathcal{A}}$ such that $\forall_{m \in \mathbb{N}}\left\{w^{m} \in B^{L-\mathcal{J}}\left(w^{0}, r\right)\right\}\left(\forall_{m \in \mathbb{N}}\left\{w^{m} \in B^{R-\mathcal{J}}\left(w^{0}, r\right)\right\}\right)$ and $\left(w^{m}: m \in\{0\} \cup \mathbb{N}\right)$ is left (right) $\mathcal{P}$-convergent to $w$.

If, moreover, $(X, T)$ is left (right) partially $\mathcal{J}$-admissible in a point $w^{0} \in X$, then the point $w$ above satisfies $w \in B^{L-\mathcal{J}}\left(w^{0}, r\right)\left(w \in B^{R-\mathcal{J}}\left(w^{0}, r\right)\right)$.

(B) If $(X, T)$ is left (right) $\mathcal{J}$-admissible in a point $w^{0} \in X$ and if, for some $s \in \mathbb{N}, T^{[s]}$ is left (right) $\mathcal{P}$-quasi-closed on $X$, then $\operatorname{Fix}\left(T^{[s]}\right) \neq \varnothing$ and there exist a dynamic process $\left(w^{m}: m \in\{0\} \cup \mathbb{N}\right)$ of the system $(X, T)$ starting at $w^{0}$, a point $w \in \operatorname{Fix}\left(T^{[s]}\right)$ and $r=\left\{r_{\alpha}\right\}_{\alpha \in \mathcal{A}} \in(0 ; \infty)^{\mathcal{A}}$ such that $\forall_{m \in \mathbb{N}}\left\{w^{m} \in B^{L-\mathcal{J}}\left(w^{0}, r\right)\right\}\left(\forall_{m \in \mathbb{N}}\left\{w^{m} \in B^{R-\mathcal{J}}\left(w^{0}, r\right)\right\}\right)$ and $\left(w^{m}: m \in\{0\} \cup \mathbb{N}\right)$ is left (right) $\mathcal{P}$-convergent to $w$.

If, moreover, $(X, T)$ is left (right) partially $\mathcal{J}$-admissible in a point $w^{0} \in X$, then the point $w$ above satisfies $w \in B^{L-\mathcal{J}}\left(w^{0}, r\right)\left(w \in B^{R-\mathcal{J}}\left(w^{0}, r\right)\right)$.

Proof We prove Theorem 10.1 only in the case when $\mathcal{J}$ is a left $\mathcal{J}$-family on $X,(X, T)$ is left $\mathcal{J}$-admissible on $X$ or left partially $\mathcal{J}$-admissible on $X$, and $T^{[s]}$ is left $\mathcal{P}$-quasi-closed map on $X$, respectively. We omit the proof in the case of 'right', which is based on an analogous technique.

Part 1. Assume that $(X, T)$ is left $\mathcal{J}$-admissible in a point $w^{0} \in X$.

By (9.1) and the fact that $J_{\alpha}: X^{2} \rightarrow[0 ; \infty), \alpha \in \mathcal{A}$, we choose

$$
r=\left\{r_{\alpha}\right\}_{\alpha \in \mathcal{A}} \in(0 ; \infty)^{\mathcal{A}}
$$


such that

$$
\forall_{\alpha \in \mathcal{A}}\left\{J_{\alpha}\left(w^{0}, T\left(w^{0}\right)\right)<\left(1-\lambda_{\alpha}\right) r_{\alpha}\right\} .
$$

Put

$$
\forall_{\alpha \in \mathcal{A}}\left\{\gamma_{\alpha}^{(0)}=\left(1-\lambda_{\alpha}\right) r_{\alpha}-J_{\alpha}\left(w^{0}, T\left(w^{0}\right)\right)\right\}
$$

In view of (10.3) and (10.4), this implies $\gamma^{(0)}=\left\{\gamma_{\alpha}^{(0)}\right\}_{\alpha \in \mathcal{A}} \in(0 ; \infty)^{\mathcal{A}}$, and we apply (10.1) to find $w^{1} \in T\left(w^{0}\right)$ such that

$$
\forall_{\alpha \in \mathcal{A}}\left\{J_{\alpha}\left(w^{0}, w^{1}\right)<J_{\alpha}\left(w^{0}, T\left(w^{0}\right)\right)+\gamma_{\alpha}^{(0)}\right\} .
$$

We see from (10.5) and (10.6) that

$$
\forall_{\alpha \in \mathcal{A}}\left\{J_{\alpha}\left(w^{0}, w^{1}\right)<\left(1-\lambda_{\alpha}\right) r_{\alpha}\right\}
$$

Observe that (10.7) implies $w^{1} \in B^{L-\mathcal{J}}\left(w^{0}, r\right)$.

Put now

$$
\forall_{\alpha \in \mathcal{A}}\left\{\gamma_{\alpha}^{(1)}=\lambda_{\alpha}\left[\left(1-\lambda_{\alpha}\right) r_{\alpha}-J_{\alpha}\left(w^{0}, w^{1}\right)\right]\right\}
$$

Then, in view of (10.7), we get $\gamma^{(1)}=\left\{\gamma_{\alpha}^{(1)}\right\}_{\alpha \in \mathcal{A}} \in(0 ; \infty)^{\mathcal{A}}$, and applying again (10.1) we find $w^{2} \in T\left(w^{1}\right)$ such that

$$
\forall_{\alpha \in \mathcal{A}}\left\{J_{\alpha}\left(w^{1}, w^{2}\right)<J_{\alpha}\left(w^{1}, T\left(w^{1}\right)\right)+\gamma_{\alpha}^{(1)}\right\} .
$$

Also note that

$$
\forall_{\alpha \in \mathcal{A}}\left\{J_{\alpha}\left(w^{1}, w^{2}\right)<\lambda_{\alpha}\left(1-\lambda_{\alpha}\right) r_{\alpha}\right\}
$$

Indeed, from (10.9), (9.1), Definition 9.1 and (10.8), we get

$$
\begin{aligned}
\forall_{\alpha \in \mathcal{A}}\left\{J_{\alpha}\left(w^{1}, w^{2}\right)<J_{\alpha}\left(w^{1}, T\left(w^{1}\right)\right)+\gamma_{\alpha}^{(1)} \leq \sup _{u \in T\left(w^{0}\right)} J_{\alpha}\left(u, T\left(w^{1}\right)\right)+\gamma_{\alpha}^{(1)}\right. \\
\quad \leq D_{\eta ; \alpha}^{L-\mathcal{J}}\left(T\left(w^{0}\right), T\left(w^{1}\right)\right)+\gamma_{\alpha}^{(1)} \leq \lambda_{\alpha} J_{\alpha}\left(w^{0}, w^{1}\right)+\gamma_{\alpha}^{(1)} \\
\left.\quad=\lambda_{\alpha}\left(1-\lambda_{\alpha}\right) r_{\alpha}\right\}, \quad \eta \in\{1,2,3\} .
\end{aligned}
$$

Thus (10.10) holds. Further, by $(\mathcal{J} 1),(10.7)$ and $(10.10)$, we observe that

$$
\forall_{\alpha \in \mathcal{A}}\left\{J_{\alpha}\left(w^{0}, x^{2}\right)<\left(1-\lambda_{\alpha}\right) r_{\alpha}\left(1+\lambda_{\alpha}\right) \leq\left(1-\lambda_{\alpha}\right) r_{\alpha} \sum_{k=0}^{\infty} \lambda_{\alpha}^{k}=r_{\alpha}\right\} .
$$

Hence $w^{2} \in B^{L-\mathcal{J}}\left(w^{0}, r\right)$. 
Proceeding as before, using Definition 9.1 and property (10.1), we get that there exists a sequence $\left(w^{m}: m \in \mathbb{N}\right)$ in $X$ satisfying

$$
\forall_{m \in \mathbb{N}}\left\{w^{m+1} \in T\left(w^{m}\right)\right\} .
$$

For calculational purposes, upon letting $\forall_{m \in \mathbb{N}}\left\{\gamma^{(m)}=\left\{\gamma_{\alpha}^{(m)}\right\}_{\alpha \in \mathcal{A}}\right\}$, where

$$
\forall_{\alpha \in \mathcal{A}} \forall_{m \in \mathbb{N}}\left\{\gamma_{\alpha}^{(m)}=\lambda_{\alpha}\left[\lambda_{\alpha}^{m-1}\left(1-\lambda_{\alpha}\right) r_{\alpha}-J_{\alpha}\left(w^{m-1}, w^{m}\right)\right]\right\},
$$

we observe that $\forall_{m \in \mathbb{N}}\left\{\gamma^{(m)} \in(0 ; \infty)^{\mathcal{A}}\right\}$,

$$
\begin{aligned}
& \forall_{\alpha \in \mathcal{A}} \forall_{m \in \mathbb{N}}\left\{J_{\alpha}\left(w^{m}, w^{m+1}\right)<J_{\alpha}\left(w^{m}, T\left(w^{m}\right)\right)+\gamma_{\alpha}^{(m)}\right\}, \\
& \forall_{\alpha \in \mathcal{A}} \forall_{m \in \mathbb{N}}\left\{J_{\alpha}\left(w^{m}, w^{m+1}\right)<\lambda_{\alpha}^{m}\left(1-\lambda_{\alpha}\right) r_{\alpha}\right\}
\end{aligned}
$$

and

$$
\forall_{\alpha \in \mathcal{A}} \forall_{m \in \mathbb{N} \cup\{0\}}\left\{J_{\alpha}\left(w^{0}, w^{m+1}\right)<\left(1-\lambda_{\alpha}\right) r_{\alpha} \sum_{k=0}^{m} \lambda_{\alpha}^{k}<\left(1-\lambda_{\alpha}\right) r_{\alpha} \sum_{k=0}^{\infty} \lambda_{\alpha}^{k}=r_{\alpha}\right\} .
$$

We see from (10.13) that $\forall_{m \in \mathbb{N}}\left\{w^{m} \in B^{L-\mathcal{J}}\left(w^{0}, r\right)\right\}$.

Let now $m>n$. Using $(\mathcal{J} 1)$ and (10.12), we get

$$
\begin{aligned}
\lim _{m \rightarrow \infty} \sup _{n>m} J_{\alpha}\left(w^{m}, w^{n}\right) & \leq \lim _{m \rightarrow \infty} \sup _{n>m} \sum_{j=m}^{n-1} J_{\alpha}\left(w^{j}, w^{j+1}\right) \leq\left(1-\lambda_{\alpha}\right) r_{\alpha} \lim _{m \rightarrow \infty} \sup _{n>m} \sum_{j=m}^{n-1} \lambda_{\alpha}^{j} \\
& \leq r_{\alpha} \lim _{m \rightarrow \infty} \lambda_{\alpha}^{m} .
\end{aligned}
$$

This means that

$$
\forall_{w_{0} \in X} \exists_{\left(w^{m}: m \in \mathbb{N}\right)} \forall_{m \in\{0\} \cup \mathbb{N}}\left\{w^{m+1} \in T\left(w^{m}\right)\right\}
$$

and

$$
\forall_{\alpha \in \mathcal{A}}\left\{\lim _{m \rightarrow \infty} \sup _{n>m} J_{\alpha}\left(w^{m}, w^{n}\right)=0\right\} .
$$

Now, since $(X, T)$ is left $\mathcal{J}$-admissible on $X$, by Definition 6.1(A), properties (10.14) and (10.15) imply that there exists $w \in X$ such that

$$
\forall \alpha \in \mathcal{A}\left\{\lim _{m \rightarrow \infty} J_{\alpha}\left(w, w^{m}\right)=0\right\}
$$

Next, defining $v_{m}=w$ and $u_{m}=w^{m}$ for $m \in \mathbb{N}$, by (10.15) and (10.16) we see that conditions (3.1) and (3.3) hold for the sequences $\left(u_{m}: m \in \mathbb{N}\right)$ and $\left(v_{m}: m \in \mathbb{N}\right)$ in $X$. Consequently, by $(\mathcal{J} 2)$ we get (3.5) which implies that

$$
\forall \forall_{\alpha \in \mathcal{A}}\left\{\lim _{m \rightarrow \infty} p_{\alpha}\left(w, w^{m}\right)=\lim _{m \rightarrow \infty} p_{\alpha}\left(v_{m}, u_{m}\right)=0\right\}
$$

and so, in particular, we see that $w \in S_{\left(w^{m}: m \in \mathbb{N}\right)}^{L-\mathcal{P}}=\left\{x \in X: \lim _{m \rightarrow \infty}^{L-\mathcal{P}} w^{m}=x\right\}$. 
Additionally, by $(\mathcal{J} 1)$ and (10.13), we note that

$$
\forall_{\alpha \in \mathcal{A}} \forall_{m \in \mathbb{N}}\left\{J_{\alpha}\left(w^{0}, w\right) \leq J_{\alpha}\left(w^{0}, w^{m}\right)+J_{\alpha}\left(w^{m}, w\right)<r_{\alpha}+J_{\alpha}\left(w^{m}, w\right)\right\} .
$$

Part 2. Assume that $(X, T)$ is left $\mathcal{J}$-admissible in a point $w^{0} \in X$ and, for some $s \in \mathbb{N}$, $T^{[s]}$ is left $\mathcal{P}$-quasi-closed on $X$.

By Part 1, $S_{\left(w^{m}: m \in\{0\} \cup \mathbb{N}\right)}^{L-\mathcal{P}} \neq \varnothing$ and since by $(10.11), w^{(m+1) s} \in T^{[s]}\left(w^{m s}\right)$ for $m \in\{0\} \cup \mathbb{N}$, thus defining $\left(x_{m}=w^{m-1+s}: m \in \mathbb{N}\right)$ we see that $\left(x_{m}: m \in \mathbb{N}\right) \subset T^{[s]}(X), S_{\left(x_{m}: m \in\{0\} \cup \mathbb{N}\right)}^{L-\mathcal{P}}=$ $S_{\left(w^{m}: m \in\{0\} \cup \mathbb{N}\right)}^{L-\mathcal{P}} \neq \varnothing$, the sequences $\left(v_{m}=w^{(m+1) s}: m \in \mathbb{N}\right) \subset T^{[s]}(X)$ and $\left(u_{m}=w^{m s}: m \in\right.$ $\mathbb{N}) \subset T^{[s]}(X)$ satisfy $\forall_{m \in \mathbb{N}}\left\{v_{m} \in T^{[s]}\left(u_{m}\right)\right\}$ and, as subsequences of $\left(x_{m}: m \in\{0\} \cup \mathbb{N}\right)$, are left $\mathcal{P}$-converging to each point of the set $S_{\left(w^{m}: m \in\{0\} \cup N\right)}^{L-\mathcal{P}}$. Moreover, by Remark 5.1(a), $S_{\left(w^{m}: m \in \mathbb{N}\right)}^{L-\mathcal{P}} \subset S_{\left(v_{m}: m \in \mathbb{N}\right)}^{L-\mathcal{P}}$ and $S_{\left(w^{m}: m \in \mathbb{N}\right)}^{L-\mathcal{P}} \subset S_{\left(u_{m}: m \in \mathbb{N}\right)}^{L-\mathcal{P}}$. By above, since $T^{[s]}$ is left $\mathcal{P}$-quasi-closed, we conclude that $\exists_{w \in S_{(w m: m \in\{0\} \cup \mathbb{N})}^{L-\mathcal{P}}}=S_{\left(x_{m}: m \in \mathbb{N}\right)}^{L-\mathcal{P}}\left\{w \in T^{[s]}(w)\right\}$.

Part 3. Assume that $(X, T)$ is left partially $\mathcal{J}$-admissible in a point $w^{0} \in X$.

Using Part 1, (10.14) and (10.15), by Definition 6.1(B), we have that there exists $w \in X$ such that

$$
\forall_{\alpha \in \mathcal{A}}\left\{\lim _{m \rightarrow \infty} J_{\alpha}\left(w^{m}, w\right)=\lim _{m \rightarrow \infty} J_{\alpha}\left(w, w^{m}\right)=0\right\} .
$$

The consequence of (10.17) and (10.18) is $w \in B^{L-\mathcal{J}}\left(w^{0}, r\right)$.

Part 4. The result now follows at once from Parts 1-3.

Theorem 10.1 and its proof immediately yields the following theorem.

Theorem 10.2 Let $(X, \mathcal{P})$ be a quasi-gauge space, let the family $\mathcal{J}=\left\{J_{\alpha}: \alpha \in \mathcal{A}\right\}$ of maps $J_{\alpha}: X^{2} \rightarrow[0 ; \infty), \alpha \in \mathcal{A}$, be a left (right) $\mathcal{J}$-family on $X$, and suppose that $\eta \in\{1,2,3\}$. Assume, moreover, that $w^{0} \in X, \lambda=\left\{\lambda_{\alpha}\right\}_{\alpha \in \mathcal{A}} \in[0 ; 1)^{\mathcal{A}}, r=\left\{r_{\alpha}\right\}_{\alpha \in \mathcal{A}} \in(0 ; \infty)^{\mathcal{A}}$ and a setvalued map

$$
T:\left\{w^{0}\right\} \cup B^{L-\mathcal{J}}\left(w^{0}, r\right) \rightarrow 2^{X} \quad\left(T:\left\{w^{0}\right\} \cup B^{R-\mathcal{J}}\left(w^{0}, r\right) \rightarrow 2^{X}\right)
$$

satisfy:

(i) $T$ is $\left(\mathcal{D}_{\eta}^{L-\mathcal{J}}, \lambda\right)$-contraction on $\left\{w^{0}\right\} \cup B^{L-\mathcal{J}}\left(w^{0}, r\right)\left(T\right.$ is $\left(\mathcal{D}_{\eta}^{R-\mathcal{J}}, \lambda\right)$-contraction on $\left.\left\{w^{0}\right\} \cup B^{R-\mathcal{J}}\left(w^{0}, r\right)\right)$

(ii) $\forall_{\alpha \in \mathcal{A}}\left\{J_{\alpha}\left(w^{0}, T\left(w^{0}\right)\right)<\left(1-\lambda_{\alpha}\right) r_{\alpha}\right\}\left(\forall_{\alpha \in \mathcal{A}}\left\{J_{\alpha}\left(T\left(w^{0}\right), w^{0}\right)<\left(1-\lambda_{\alpha}\right) r_{\alpha}\right\}\right)$; and

(iii) for every $x \in\left\{w^{0}\right\} \cup B^{L-\mathcal{J}}\left(w^{0}, r\right)\left(x \in\left\{w^{0}\right\} \cup B^{R-\mathcal{J}}\left(w^{0}, r\right)\right)$ and for every $\gamma=\left\{\gamma_{\alpha}\right\}_{\alpha \in \mathcal{A}} \in(0 ; \infty)^{\mathcal{A}}$, there exists $y \in T(x)$ such that

$$
\forall_{\alpha \in \mathcal{A}}\left\{J_{\alpha}(x, y)<J_{\alpha}(x, T(x))+\gamma_{\alpha}\right\} \quad\left(\forall_{\alpha \in \mathcal{A}}\left\{J_{\alpha}(y, x)<J_{\alpha}(T(x), x)+\gamma_{\alpha}\right\}\right) .
$$

(A) If $T$ is left (right) partially $\mathcal{J}$-admissible in $w^{0}$, then there exist a dynamic process $\left(w^{m}: m \in\{0\} \cup \mathbb{N}\right)$ of the system $(X, T)$ starting at $w^{0}$ and a point $w \in B^{L-\mathcal{J}}\left(w^{0}, r\right)$ $\left(w \in B^{R-\mathcal{J}}\left(w^{0}, r\right)\right)$ such that $\forall_{m \in \mathbb{N}}\left\{w^{m} \in B^{L-\mathcal{J}}\left(w^{0}, r\right)\right\}\left(\forall_{m \in \mathbb{N}}\left\{w^{m} \in B^{R-\mathcal{J}}\left(w^{0}, r\right)\right\}\right)$ and $\left(w^{m}: m \in\{0\} \cup \mathbb{N}\right)$ is left (right) $\mathcal{P}$-convergent to $w$.

(B) If $T$ is left (right) partially $\mathcal{J}$-admissible in $w^{0}$ and if $T$ is left (right) $\mathcal{P}$-quasi-closed on $\left\{w^{0}\right\} \cup B^{L-\mathcal{J}}\left(w^{0}, r\right)\left(\right.$ on $\left.\left\{w^{0}\right\} \cup B^{R-\mathcal{J}}\left(w^{0}, r\right)\right)$, then $\operatorname{Fix}(T) \neq \varnothing$ and there exist a 
dynamic process $\left(w^{m}: m \in\{0\} \cup \mathbb{N}\right)$ of the system $(X, T)$ starting at $w^{0}$ and a point $w \in \operatorname{Fix}(T)$ such that $\forall_{m \in \mathbb{N}}\left\{w^{m} \in B^{L-\mathcal{J}}\left(w^{0}, r\right)\right\}\left(\forall_{m \in \mathbb{N}}\left\{w^{m} \in B^{R-\mathcal{J}}\left(w^{0}, r\right)\right\}\right)$, $\left(w^{m}: m \in\{0\} \cup \mathbb{N}\right)$ is left (right) $\mathcal{P}$-convergent to $w$ and $w \in B^{L-\mathcal{J}}\left(w^{0}, r\right)$ $\left(w \in B^{R-\mathcal{J}}\left(w^{0}, r\right)\right)$.

\section{Convergence, existence, periodic point, fixed point, localization and uniqueness results for single-valued left (right) contractions of Banach type}

In this section we indicate how to extend the results of the preceding section to singlevalued maps.

Definition 11.1 Let $(X, \mathcal{P})$ be a quasi-gauge space, let the family $\mathcal{J}=\left\{J_{\alpha}: \alpha \in \mathcal{A}\right\}$ of maps $J_{\alpha}: X^{2} \rightarrow[0 ; \infty), \alpha \in \mathcal{A}$, be a left (right) $\mathcal{J}$-family on $X$, and let $\eta \in\{1,2\}$.

(A) Define on $X$ the left (right) distance $\mathcal{D}_{\eta}^{L-\mathcal{J}}=\left\{D_{\eta ; \alpha}^{L-\mathcal{J}}: X^{2} \rightarrow[0 ; \infty), \alpha \in \mathcal{A}\right\}$ $\left(\mathcal{D}_{\eta}^{R-\mathcal{J}}=\left\{D_{\eta ; \alpha}^{R-\mathcal{J}}: X^{2} \rightarrow[0 ; \infty), \alpha \in \mathcal{A}\right\}\right)$ as follows:

(A.1) $\forall_{\alpha \in \mathcal{A}} \forall_{u, v \in X}\left\{D_{1 ; \alpha}^{L-\mathcal{J}}(u, v)=\max \left\{J_{\alpha}(u, v), J_{\alpha}(v, u)\right\}\right\}$ and $\forall_{\alpha \in \mathcal{A}} \forall_{u, v \in X}\left\{D_{2 ; \alpha}^{L-\mathcal{J}}(u, v)=\right.$ $\left.J_{\alpha}(u, v)\right\}$ if $\mathcal{J} \in \mathbb{J}_{(X, \mathcal{P})}^{L}$;

(A.2) $\forall_{\alpha \in \mathcal{A}} \forall_{u, v \in X}\left\{D_{1 ; \alpha}^{R-\mathcal{J}}(u, v)=\max \left\{J_{\alpha}(u, v), J_{\alpha}(v, u)\right\}\right\}$ and $\forall_{\alpha \in \mathcal{A}} \forall_{u, v \in X}\left\{D_{2 ; \alpha}^{R-\mathcal{J}}(u, v)=\right.$ $\left.J_{\alpha}(u, v)\right\}$ if $\mathcal{J} \in \mathbb{J}_{(X, \mathcal{P})}^{R}$.

(B) Let $\lambda=\left\{\lambda_{\alpha}\right\}_{\alpha \in \mathcal{A}} \in[0 ; 1)^{\mathcal{A}}$ and let $Y \in 2^{X}$. We say that a single-valued map

$T: Y \rightarrow X$ is $\left(\mathcal{D}_{\eta}^{L-\mathcal{J}}, \lambda\right)$-contraction on $Y\left(\left(\mathcal{D}_{\eta}^{R-\mathcal{J}}, \lambda\right)\right.$-contraction on $\left.Y\right)$ if:

(B.1) $\forall_{\alpha \in \mathcal{A}} \forall_{x, y \in Y}\left\{D_{\eta ; \alpha}^{L-\mathcal{J}}(T(x), T(y)) \leq \lambda_{\alpha} J_{\alpha}(x, y)\right\}$ if $\mathcal{J} \in \mathbb{J}_{(X, \mathcal{P})}^{L}$;

(B.2) $\forall_{\alpha \in \mathcal{A}} \forall_{x, y \in Y}\left\{D_{\eta ; \alpha}^{R-\mathcal{J}}(T(x), T(y)) \leq \lambda_{\alpha} J_{\alpha}(x, y)\right\}$ if $\mathcal{J} \in \mathbb{J}_{(X, \mathcal{P})}^{R}$.

As a consequence of Definition 11.1 and Theorems 10.1 and 10.2, we have the following results.

Theorem 11.1 Let $(X, \mathcal{P})$ be a quasi-gauge space, let the family $\mathcal{J}=\left\{J_{\alpha}: \alpha \in \mathcal{A}\right\}$ of maps $J_{\alpha}: X^{2} \rightarrow[0 ; \infty), \alpha \in \mathcal{A}$, be a left (right) $\mathcal{J}$-family on $X$, and suppose that $\eta \in\{1,2\}$. Let $\lambda=$ $\left\{\lambda_{\alpha}\right\}_{\alpha \in \mathcal{A}} \in[0 ; 1)^{\mathcal{A}}$ and let a single-valued dynamic system $(X, T), T: X \rightarrow X$, be $\left(\mathcal{D}_{\eta}^{L-\mathcal{J}}, \lambda\right)$ contraction on $X\left(\left(\mathcal{D}_{\eta}^{R-\mathcal{J}}, \lambda\right)\right.$-contraction on $\left.X\right)$.

(A) If $(X, T)$ is left (right) $\mathcal{J}$-admissible in a point $w^{0} \in X$, then there exist a point $w \in X$ and $r=\left\{r_{\alpha}\right\}_{\alpha \in \mathcal{A}} \in(0 ; \infty)^{\mathcal{A}}$ such that the sequence $\left(w^{m}=T^{[m]}\left(w^{0}\right): m \in\{0\} \cup \mathbb{N}\right)$ is left (right) $\mathcal{P}$-convergent to $w$ and $\forall_{m \in \mathbb{N}}\left\{w^{m} \in B^{L-\mathcal{J}}\left(w^{0}, r\right)\right\}$

$\left(\forall_{m \in \mathbb{N}}\left\{w^{m} \in B^{R-\mathcal{J}}\left(w^{0}, r\right)\right\}\right)$.

If, moreover, $(X, T)$ is left (right) partially $\mathcal{J}$-admissible in a point $w^{0} \in X$, then the point $w$ above satisfies $w \in B^{L-\mathcal{J}}\left(w^{0}, r\right)\left(w \in B^{R-\mathcal{J}}\left(w^{0}, r\right)\right)$.

(B) If $(X, T)$ is left (right) $\mathcal{J}$-admissible in a point $w^{0} \in X$ and if, for some $s \in \mathbb{N}, T^{[s]}$ is left (right) $\mathcal{P}$-quasi-closed on $X$, then $\operatorname{Fix}\left(T^{[s]}\right) \neq \varnothing$ and there exist a point $w \in \operatorname{Fix}\left(T^{[s]}\right)$ and $r=\left\{r_{\alpha}\right\}_{\alpha \in \mathcal{A}} \in(0 ; \infty)^{\mathcal{A}}$ such that the sequence $\left(w^{m}=T^{[m]}\left(w^{0}\right): m \in\{0\} \cup \mathbb{N}\right)$ is left (right) $\mathcal{P}$-convergent to $w$, $\forall_{m \in \mathbb{N}}\left\{w^{m} \in B^{L-\mathcal{J}}\left(w^{0}, r\right)\right\}\left(\forall_{m \in \mathbb{N}}\left\{w^{m} \in B^{R-\mathcal{J}}\left(w^{0}, r\right)\right\}\right)$ and

$$
\forall_{\alpha \in \mathcal{A}} \forall_{v \in \operatorname{Fix}\left(T^{[s]}\right)}\left\{J_{\alpha}(v, T(v))=J_{\alpha}(T(v), v)=0\right\} .
$$

If, moreover, $(X, T)$ is left (right) partially $\mathcal{J}$-admissible in a point $w^{0} \in X$, then the point $w$ above satisfies $w \in B^{L-\mathcal{J}}\left(w^{0}, r\right)\left(w \in B^{R-\mathcal{J}}\left(w^{0}, r\right)\right)$. 
(C) If $(X, T)$ is left (right) $\mathcal{J}$-admissible in a point $w^{0} \in X$, if, for some $s \in \mathbb{N}, T^{[s]}$ is left (right) $\mathcal{P}$-quasi-closed on $X$ and if $(X, \mathcal{P})$ is a Hausdorff space, then there exists a point $w \in X$ such that $\operatorname{Fix}\left(T^{[s]}\right)=\operatorname{Fix}(T)=\{w\}$, the sequence $\left(w^{m}=T^{[m]}\left(w^{0}\right): m \in\{0\} \cup \mathbb{N}\right)$ is left (right) $\mathcal{P}$-convergent to $w$, $\forall_{m \in \mathbb{N}}\left\{w^{m} \in B^{L-\mathcal{J}}\left(w^{0}, r\right)\right\}\left(\forall_{m \in \mathbb{N}}\left\{w^{m} \in B^{R-\mathcal{J}}\left(w^{0}, r\right)\right\}\right)$ and

$$
\forall_{\alpha \in \mathcal{A}}\left\{J_{\alpha}(w, w)=0\right\} .
$$

If, moreover, $(X, T)$ is left (right) partially $\mathcal{J}$-admissible in a point $w^{0} \in X$, then the point $w$ above satisfies $w \in B^{L-\mathcal{J}}\left(w^{0}, r\right)\left(w \in B^{R-\mathcal{J}}\left(w^{0}, r\right)\right)$.

Proof We prove only (11.1) and (11.2) and only in the case when $\mathcal{J}$ is a left $\mathcal{J}$-family on $X$, $(X, T)$ is left $\mathcal{J}$-admissible in $w^{0}$ or left partially $\mathcal{J}$-admissible in $w^{0}$, and $T^{[s]}$ is left $\mathcal{P}$ closed map on $X$, respectively. We omit the proof in the case of 'right', which is based on an analogous technique.

Part 1. Property (11.1) holds.

Indeed, first suppose that $\exists_{\alpha_{0} \in \mathcal{A}} \exists_{v \in \operatorname{Fix}\left(T^{[s]}\right)}\left\{J_{\alpha_{0}}(v, T(v))>0\right\}$. Of course, $v=T^{[2 s]}(v), T(v)=$ $T^{[2 s]}(T(v))$ and, for $\eta \in\{1,2\}$, by Definition 11.1, $0<J_{\alpha_{0}}(v, T(v))=J_{\alpha_{0}}\left(T^{[2 s]}(v), T^{[2 s]}(T(v))\right) \leq$ $D_{\eta ; \alpha_{0}}^{L-\mathcal{J}}\left(T^{[2 s]}(v), T^{[2 s]}(T(v))\right) \leq \lambda_{\alpha_{0}} J_{\alpha_{0}}\left(T^{[2 s-1]}(v), T^{[2 s-1]}(T(v))\right) \leq \lambda_{\alpha_{0}} D_{\eta ; \alpha_{0}}^{L-\mathcal{J}}\left(T^{[2 s-1]}(v)\right.$, $\left.T^{[2 s-1]}(T(v))\right) \leq \lambda_{\alpha_{0}}^{2} J_{\alpha_{0}}\left(T^{[2 s-2]}(v), T^{[2 s-2]}(T(v))\right) \leq \cdots \leq \lambda_{\alpha_{0}}^{2 s} J_{\alpha_{0}}(v, T(v))<J_{\alpha_{0}}(v, T(v))$, which is impossible.

Suppose now that $\exists_{\alpha_{0} \in \mathcal{A}} \exists_{v \in \operatorname{Fix}\left(T^{[s]}\right)}\left\{J_{\alpha_{0}}(T(v), v)>0\right\}$. Then, by Definition 11.1, using the fact that $v=T^{[s]}(v)=T^{[2 s]}(v)$, we get, for $\eta \in\{1,2\}, 0<J_{\alpha_{0}}(T(v), v)=J_{\alpha_{0}}\left(T^{[s+1]}(v), T^{[2 s]}(v)\right) \leq$ $\sum_{k=s+1}^{2 s-1} J_{\alpha_{0}}\left(T^{[k]}(v), T^{[k+1]}(v)\right) \leq \sum_{k=s+1}^{2 s-1} D_{\eta ; \alpha_{0}}^{L-\mathcal{J}}\left(T^{[k]}(v), T^{[k+1]}(v)\right) \leq \sum_{k=s+1}^{2 s-1} \lambda_{\alpha_{0}}^{k} J_{\alpha_{0}}(v, T(v))=0$, which is impossible.

Therefore, (11.1) holds.

Part 2. Property (11.2) holds.

If $(X, \mathcal{P})$ is a Hausdorff space, then Remark 3.1(d) and property (11.1) imply $\forall_{v \in \operatorname{Fix}\left(T^{[s]}\right)}\{T(v)=v\}$ and $\forall_{\alpha \in \mathcal{A}} \forall_{v \in \operatorname{Fix}\left(T^{[s]}\right)}\left\{J_{\alpha}(v, v) \leq J_{\alpha}(v, T(v))+J_{\alpha}(T(v), v)=0\right\}$. Therefore, $\operatorname{Fix}\left(T^{[s]}\right)=\operatorname{Fix}(T)$ and

$$
\forall_{v \in \operatorname{Fix}\left(T^{[s]}\right)=\operatorname{Fix}(T)}\left\{J_{\alpha}(v, v)=0\right\} .
$$

Suppose now that $u, w \in \operatorname{Fix}(T)$ and $u \neq w$. Then, by Remark 3.1(d), $\exists_{\alpha_{0} \in \mathcal{A}}\left\{J_{\alpha_{0}}(u, w)>\right.$ $\left.0 \vee J_{\alpha_{0}}(w, u)>0\right\}$. Of course, for $\eta \in\{1,2\}$, we then have $\exists_{\alpha_{0} \in \mathcal{A}}\left\{U_{\alpha_{0}}(u, w)>0 \wedge J_{\alpha_{0}}(u, w)=\right.$ $\left.J_{\alpha_{0}}(T(u), T(w)) \leq D_{\eta ; \alpha_{0}}^{L-\mathcal{J}}(T(u), T(w)) \leq \lambda_{\alpha_{0}} J_{\alpha_{0}}(u, w)<J_{\alpha_{0}}(u, w)\right] \vee\left[J_{\alpha_{0}}(w, u)>0 \wedge J_{\alpha_{0}}(w, u)=\right.$ $\left.\left.J_{\alpha_{0}}(T(w), T(u)) \leq D_{\eta ; \alpha_{0}}^{L-\mathcal{J}}(T(w), T(u)) \leq \lambda_{\alpha_{0}} J_{\alpha_{0}}(w, u)<J_{\alpha_{0}}(w, u)\right]\right\}$, which is impossible. This gives that $\operatorname{Fix}(T)$ is a singleton.

Therefore, (11.2) holds.

Theorem 11.2 Let $(X, \mathcal{P})$ be a quasi-gauge space, let the family $\mathcal{J}=\left\{J_{\alpha}: \alpha \in \mathcal{A}\right\}$ of maps $J_{\alpha}: X^{2} \rightarrow[0 ; \infty), \alpha \in \mathcal{A}$, be a left (right) $\mathcal{J}$-family on $X$, and suppose that $\eta \in\{1,2\}$. Let $w^{0} \in X, \lambda=\left\{\lambda_{\alpha}\right\}_{\alpha \in \mathcal{A}} \in[0 ; 1)^{\mathcal{A}}, r=\left\{r_{\alpha}\right\}_{\alpha \in \mathcal{A}} \in(0 ; \infty)^{\mathcal{A}}$ and a single-valued map $T:\left\{w^{0}\right\} \cup$ $B^{L-\mathcal{J}}\left(w^{0}, r\right) \rightarrow X\left(T:\left\{w^{0}\right\} \cup B^{R-\mathcal{J}}\left(w^{0}, r\right) \rightarrow X\right)$ be such that

$$
T \text { is }\left(\mathcal{D}_{\eta}^{L-\mathcal{J}}, \lambda\right) \text {-contraction on }\left\{w^{0}\right\} \cup B^{L-\mathcal{J}}\left(w^{0}, r\right)
$$


$\left(T\right.$ is $\left(\mathcal{D}_{\eta}^{R-\mathcal{J}}, \lambda\right)$-contraction on $\left.\left\{w^{0}\right\} \cup B^{R-\mathcal{J}}\left(w^{0}, r\right)\right)$

and $\forall_{\alpha \in \mathcal{A}}\left\{J_{\alpha}\left(w^{0}, T\left(w^{0}\right)\right)<\left(1-\lambda_{\alpha}\right) r_{\alpha}\right\}\left(\forall_{\alpha \in \mathcal{A}}\left\{J_{\alpha}\left(T\left(w^{0}\right), w^{0}\right)<\left(1-\lambda_{\alpha}\right) r_{\alpha}\right\}\right)$.

(A) If $T$ is left (right) partially $\mathcal{J}$-admissible in $w^{0}$, then there exists a point $w \in X$ such that the sequence $\left(w^{m}=T^{[m]}\left(w^{0}\right): m \in\{0\} \cup \mathbb{N}\right)$ is left (right) $\mathcal{P}$-convergent to $w$, $\forall_{m \in \mathbb{N}}\left\{w^{m} \in B^{L-\mathcal{J}}\left(w^{0}, r\right)\right\}\left(\forall_{m \in \mathbb{N}}\left\{w^{m} \in B^{R-\mathcal{J}}\left(w^{0}, r\right)\right\}\right)$ and $w \in B^{L-\mathcal{J}}\left(w^{0}, r\right)$ $\left(w \in B^{R-\mathcal{J}}\left(w^{0}, r\right)\right)$.

(B) If $T$ is left (right) partially $\mathcal{J}$-admissible in $w^{0}$ and if $T$ is left (right) $\mathcal{P}$-quasi-closed on $B^{L-\mathcal{J}}\left(w^{0}, r\right)\left(B^{R-\mathcal{J}}\left(w^{0}, r\right)\right)$, then $\operatorname{Fix}(T) \neq \varnothing$, and there exists a point $w \in \operatorname{Fix}(T)$ such that the sequence $\left(w^{m}=T^{[m]}\left(w^{0}\right): m \in\{0\} \cup \mathbb{N}\right)$ is left (right) $\mathcal{P}$-convergent to $w, \forall_{m \in \mathbb{N}}\left\{w^{m} \in B^{L-\mathcal{J}}\left(w^{0}, r\right)\right\}\left(\forall_{m \in \mathbb{N}}\left\{w^{m} \in B^{R-\mathcal{J}}\left(w^{0}, r\right)\right\}\right), w \in B^{L-\mathcal{J}}\left(w^{0}, r\right)$ $\left(w \in B^{R-\mathcal{J}}\left(w^{0}, r\right)\right)$ and

$$
\forall_{\alpha \in \mathcal{A}} \forall_{v \in \operatorname{Fix}(T)}\left\{J_{\alpha}(v, T(v))=J_{\alpha}(T(v), v)=0\right\} .
$$

(C) If $T$ is left (right) partially $\mathcal{J}$-admissible in $w^{0}$, if $T$ is left (right) $\mathcal{P}$-quasi-closed on $B^{L-\mathcal{J}}\left(w^{0}, r\right)\left(B^{R-\mathcal{J}}\left(w^{0}, r\right)\right)$ and if $(X, \mathcal{P})$ is a Hausdorff space, then there exists $w \in X$ such that $\operatorname{Fix}(T)=\{w\}$, the sequence $\left(w^{m}=T^{[m]}\left(w^{0}\right): m \in\{0\} \cup \mathbb{N}\right)$ is left (right)

$\mathcal{P}$-convergent to $w, \forall_{m \in \mathbb{N}}\left\{w^{m} \in B^{L-\mathcal{J}}\left(w^{0}, r\right)\right\}\left(\forall_{m \in \mathbb{N}}\left\{w^{m} \in B^{R-\mathcal{J}}\left(w^{0}, r\right)\right\}\right)$, $w \in B^{L-\mathcal{J}}\left(w^{0}, r\right)\left(w \in B^{R-\mathcal{J}}\left(w^{0}, r\right)\right)$ and

$$
\forall_{\alpha \in \mathcal{A}}\left\{J_{\alpha}(w, w)=0\right\} .
$$

\section{Examples illustrating Theorem 10.1 and comparison of Theorem 10.1 with} Theorem 1.2 of Nadler

Example 12.1 Let $(X, d), X=[0 ; 6]$, be a metric space with a metric $d: X^{2} \rightarrow[0 ; \infty)$ of the form $d(x, y)=|x-y|, x, y \in X$, and let $\mathcal{P}=\{d\}$. Let $T: X \rightarrow 2^{X}$ be of the form

$$
T(x)=\left\{\begin{array}{ll}
{[1 ; 2]} & \text { for } x \in(0 ; 3) \cup(3 ; 6), \\
{[1 ; 2] \cup[4 ; 5]} & \text { for } x=3, \\
{[4 ; 5]} & \text { for } x \in\{0,6\},
\end{array} \quad x \in X .\right.
$$

Let $E=(0 ; 3) \cup(3 ; 6)$ and let $\mathcal{J}=\{J\}$, where $J$ is of the form

$$
J(x, y)=\left\{\begin{array}{ll}
d(x, y) & \text { if } E \cap\{x, y\}=\{x, y\}, \\
7 & \text { if } E \cap\{x, y\} \neq\{x, y\},
\end{array} \quad x, y \in X\right.
$$

(I.1) $(X, \mathcal{P})$ is a quasi-gauge space and $(X, \mathcal{P})$ is left and right $\mathcal{P}$-sequentially complete.

(I.2) The property $T: X \rightarrow C l^{L-\mathcal{P}}(X)=C l^{R-\mathcal{P}}(X)$ holds.

(I.3) $J$ is symmetric and $\mathcal{J}=\{J\} \in \mathbb{J}_{(X, \mathcal{P})}^{L} \cap \mathbb{J}_{(X, \mathcal{P})}^{R}$. See Example 4.1.

(I.4) The set-valued dynamic system $(X, T)$ is a $\left(\mathcal{D}_{1}^{L-\mathcal{J}}=\mathcal{D}_{1}^{R-\mathcal{J}}, \lambda=3 / 7\right)$-contraction on $X$, i.e., $\forall_{x, y \in X}\left\{D_{1}^{L-\mathcal{J}}(T(x), T(y)) \leq(3 / 7) J(x, y)\right\}$, where

$$
D_{1}^{L-\mathcal{J}}(U, V)=D_{1}^{R-\mathcal{J}}(U, V)=\max \left\{\sup _{u \in U} J(u, V), \sup _{v \in V} J(U, v)\right\}, \quad U, V \in 2^{X} .
$$

Indeed, denoting $D_{1}^{L-\mathcal{J}}=D_{1}^{R-\mathcal{J}}=D_{1}$, we see that this follows from (I.1)-(I.3) and from Cases I.4.1-I.4.6 below. 
Case I.4.1. If $x, y \in(0 ; 3) \cup(3 ; 6)$, then $x, y \in E$ and $T(x)=T(y)=[1 ; 2] \subset E$. Hence, $\forall_{u \in T(x)=[1 ; 2]}\{\inf \{J(u, v): v \in T(y)=[1 ; 2]\}=d(u, u)=0\}$ and, consequently, $D_{1}(T(x), T(y))=$ $0 \leq \lambda J(x, y)$.

Case I.4.2. If $x \in(0 ; 3) \cup(3 ; 6)$ and $y=3$, then $x \in E, y \notin E, J(x, y)=7, T(x)=[1 ; 2] \subset$ $E, T(y)=[1 ; 2] \cup[4 ; 5] \subset E, \sup _{u \in T(x)} J(u, T(y))=0$ since $\forall_{u \in T(x)=[1 ; 2]}\{\inf U(u, v): v \in T(y)=$ $[1 ; 2] \cup[4 ; 5]\}=d(u, u)=0\}$, and $\sup _{v \in T(y)} J(T(x), v)=3$ since $v \in T(y)=[1 ; 2] \cup[4 ; 5]$ implies

$$
\inf \{J(u, v): u \in T(x)=[1 ; 2]\}= \begin{cases}d(v, v)=0 & \text { if } v \in[1 ; 2] \\ d(2, v)=v-2 & \text { if } v \in[4 ; 5]\end{cases}
$$

Therefore, $D_{1}(T(x), T(y))=3=\lambda J(x, y)$.

Case I.4.3. If $x \in(0 ; 3) \cup(3 ; 6)$ and $y \in\{0,6\}$, then $x \in E, y \notin E, T(x)=[1 ; 2] \subset$ $E, T(y)=[4 ; 5] \subset E$ and $J(x, y)=7$. We calculate: (a) If $u \in T(x)$, then $J(u, T(y))=$ $d(u,[4 ; 5])=4-u$ and, consequently, $\sup \{J(u, T(y)): u \in T(x)\}=3$; (b) If $v \in T(y)$, then $J(T(x), v)=d([1 ; 2], v)=v-2$ and, consequently, $\sup \{(T(x), v): v \in T(y)\}=3$; (c) Inequality $D_{1}(T(x), T(y))=3=\lambda 7=\lambda J(x, y)$ is a consequence of (a) and (b).

Case I.4.4. If $x=3$ and $y \in\{0,6\}$, then $x \notin E, y \notin E, T(x)=[1 ; 2] \cup[4 ; 5] \subset E, T(y)=$ $[4 ; 5] \subset E$ and $J(x, y)=7$. We calculate: (a) If $u \in T(x)$, then

$$
J(u, T(y))=d(u,[4 ; 5])= \begin{cases}4-u & \text { if } u \in[1 ; 2] \\ 0 & \text { if } u \in[4 ; 5]\end{cases}
$$

and, consequently, $\sup \{(u, T(y)): u \in T(x)\}=3$; (b) If $v \in T(y)$, then $J(T(x), v)=d(T(x)$, $v)=0$; (c) The consequence of (a) and (b) is that $D_{1}(T(x), T(y))=3=\lambda 7=\lambda J(x, y)$.

Case I.4.5. If $x=y=3$, then $x \notin E, y \notin E, T(x)=T(y)=[1 ; 2] \cup[4 ; 5] \subset E, J(u, T(y))=$ $J(T(x), v)=0$ for $u \in T(x)$ and $v \in T(y), J(x, y)=7$ and $D_{1}(T(x), T(y))=0<\lambda J(x, y)$.

Case I.4.6. If $x, y \in\{0,6\}$, then $x \notin E, y \notin E, T(x)=T(y)=[4 ; 5] \subset E, J(u, T(y))=$ $J(T(x), v)=0$ for $u \in T(x)$ and $v \in T(y), J(x, y)=7$ and $D_{1}(T(x), T(y))=0<\lambda J(x, y)$.

(I.5) Property (10.1) holds, i.e., $\forall_{x \in X} \forall_{\gamma \in(0 ; \infty)} \exists_{y \in T x}\{J(x, y)<J(x, T(x))+\gamma\}$. Indeed, this follows from Cases I.5.1-I.5.4 below.

Case 1.5.1. If $x_{0} \in\{0,3,6\}$ and $y_{0} \in T\left(x_{0}\right) \subset E$, then, since $T\left(x_{0}\right) \subset E, J\left(x_{0}, y_{0}\right)=$ $J\left(x_{0}, T\left(x_{0}\right)\right)=7$. Hence, $\forall_{\gamma \in(0 ; \infty)}\left\{J\left(x_{0}, y_{0}\right)<J\left(x_{0}, T\left(x_{0}\right)\right)+\gamma\right\}$.

Case I.5.2. If $x_{0} \in(0 ; 1]$ and $y_{0}=1 \in T\left(x_{0}\right)=[1 ; 2]$, then $J\left(x_{0}, y_{0}\right)=J\left(x_{0}, T\left(x_{0}\right)\right)=1-x_{0}$ and $\forall_{\gamma \in(0 ; \infty)}\left\{J\left(x_{0}, y_{0}\right)<J\left(x_{0}, T\left(x_{0}\right)\right)+\gamma\right\}$.

Case I.5.3. If $x_{0} \in(1 ; 2]$ and $y_{0}=x_{0} \in T\left(x_{0}\right)=[1 ; 2]$, then $J\left(x_{0}, y_{0}\right)=J\left(x_{0}, T\left(x_{0}\right)\right)=0$ and $\forall_{\gamma \in(0 ; \infty)}\left\{J\left(x_{0}, y_{0}\right)<J\left(x_{0}, T\left(x_{0}\right)\right)+\gamma\right\}$.

Case I.5.4. If $x_{0} \in(2 ; 3) \cup(3 ; 6)$ and $y_{0}=2 \in T\left(x_{0}\right)=[1 ; 2]$, then $J\left(x_{0}, y_{0}\right)=J\left(x_{0}, T\left(x_{0}\right)\right)=$ $x_{0}-2$ and $\forall_{\gamma \in(0 ; \infty)}\left\{J\left(x_{0}, y_{0}\right)<J\left(x_{0}, T\left(x_{0}\right)\right)+\gamma\right\}$.

(I.6) The set-valued dynamic system $(X, T)$ is partially $\mathcal{J}$-admissible in $X$. In fact, observing that $T^{[m]}(X)=[1 ; 2] \subset E$ for $m \geq 2$, it remains to verify that if $w^{0} \in X$ and ( $w^{m}$ : $m \in\{0\} \cup \mathbb{N}$ ) are such that $\forall_{m \in\{0\} \cup \mathbb{N}}\left\{w^{m+1} \in T\left(w^{m}\right)\right\}$ and $\lim _{m \rightarrow \infty} \sup _{n>m} J\left(w^{m}, w^{n}\right)=0$, then $\exists_{w \in[1 ; 2] \subset X}\left\{\lim _{m \rightarrow \infty} J\left(w^{m}, w\right)=\lim _{m \rightarrow \infty} J\left(w, w^{m}\right)=0\right\}$. One way to check this is as follows: We see that $\exists_{m_{1} \in \mathbb{N}} \forall_{m \geq m_{1}}\left\{w^{m} \in[1 ; 2] \subset E\right\}$ and, in view of $(12.2), \lim _{m \rightarrow \infty} \sup _{n>m} J\left(w^{m}, w^{n}\right)=$ 0 implies $\lim _{m \rightarrow \infty} \sup _{n>m} d\left(w^{m}, w^{n}\right)=0$. Moreover, [1;2] $\in C l^{L-\mathcal{P}}(X)=C l^{R-\mathcal{P}}(X)$. From this information we deduce that $\exists_{w \in[1 ; 2] \subset X}\left\{\lim _{m \rightarrow \infty} d\left(w^{m}, w\right)=\lim _{m \rightarrow \infty} J\left(w^{m}, w\right)=\right.$ $\left.\lim _{m \rightarrow \infty} J\left(w, w^{m}\right)=0\right\}$. 
(I.7) The set-valued dynamic system $(X, T)$ is a left and right $\mathcal{P}$-quasi-closed map in $X$. Indeed, let $\left(x_{m}: m \in \mathbb{N}\right) \subset T(X)=[1 ; 2] \cup[4 ; 5]$ be a left (thus also right) $\mathcal{P}$-converging sequence in $X$. Since $T(X) \in C l^{L-\mathcal{P}}(X)=C l^{R-\mathcal{P}}(X)$, thus $\exists_{x \in T(X)}\left\{\lim _{m \rightarrow \infty} d\left(x_{m}, x\right)=0\right\}$. Then we remark that the following two cases hold.

Case I.7.1. If $x \in[1 ; 2]$, then $\exists_{m_{1} \in \mathbb{N}} \forall_{m \geq m_{1}}\left\{x_{m} \in[1 ; 2]\right\}$ and if $\left(v_{m}: m \in \mathbb{N}\right)$ and $\left(u_{m}\right.$ : $m \in \mathbb{N})$ are subsequences of $\left(x_{m}: m \in \mathbb{N}\right)$ satisfying $\forall_{m \in \mathbb{N}}\left\{v_{m} \in T\left(u_{m}\right)\right\}$, then we get $\exists_{m_{2} \in \mathbb{N}} \forall_{m \geq m_{2}}\left\{u_{m} \in[1 ; 2] \wedge v_{m} \in T\left(u_{m}\right)=[1 ; 2]\right\}$. Moreover, $x \in T(x)$.

Case I.7.2. Suppose now that $x \in[4 ; 5]$. Then $\exists_{m_{1} \in \mathbb{N}} \forall_{m \geq m_{1}}\left\{x_{m} \in[4 ; 5]\right\}$ and if $\left(v_{m}: m \in \mathbb{N}\right)$ and $\left(u_{m}: m \in \mathbb{N}\right)$ are subsequences of $\left(x_{m}: m \in \mathbb{N}\right)$ satisfying $\forall_{m \in \mathbb{N}}\left\{v_{m} \in T\left(u_{m}\right)\right\}$, then we get $\exists_{m_{2} \in \mathbb{N}} \forall_{m \geq m_{2}}\left\{u_{m}, v_{m} \in[4 ; 5] \wedge v_{m} \in T\left(u_{m}\right)=[1 ; 2]\right\}$, which is impossible. Let us observe, additionally, that then also $x \notin T(x)$.

(I.8) All the assumptions of Theorem 10.1 are satisfied. This follows from (I.1)-(I.7).

We conclude that $\operatorname{Fix}(T)=[1 ; 2]$ and one shows the following.

Claim I.8.1. If $w^{0} \in\{0,3,6\}$ and $w \in[1 ; 2]$ are arbitrary and fixed, then defining $w^{m}=w$ for $m \in \mathbb{N}$ we get that $w \in T(w), \forall_{m \in \mathbb{N}}\left\{w^{m} \in B^{L-\mathcal{J}}\left(w^{0}, 7\right)\right\}$ and $\lim _{m \rightarrow \infty} d\left(w^{m}, w\right)=0$.

Claim I.8.2. If $w^{0} \in(0 ; 2)$ and $w \in[1 ; 2]$ are arbitrary and fixed, then defining $w^{m}=w$ for $m \in \mathbb{N}$ we get that $w \in T(w), \forall_{m \in \mathbb{N}}\left\{w^{m} \in B^{L-\mathcal{J}}\left(w^{0},\left|w-w^{0}\right|\right)\right\}$ and $\lim _{m \rightarrow \infty} d\left(w^{m}, w\right)=0$.

Claim I.8.3. If $w^{0} \in[2 ; 3)$ and $w \in[1 ; 2)$ are arbitrary and fixed, then defining $w^{m}=w$ for $m \in \mathbb{N}$ we get that $w \in T(w), \forall_{m \in \mathbb{N}}\left\{w^{m} \in B^{L-\mathcal{J}}\left(w^{0}, w^{0}-w\right)\right\}$ and $\lim _{m \rightarrow \infty} d\left(w^{m}, w\right)=0$.

Claim I.8.4. If $w^{0} \in(3 ; 6)$ and $w \in[1 ; 2]$ are arbitrary and fixed, then defining $w^{m}=w$ for $m \in \mathbb{N}$ we get that $w \in T(w), \forall_{m \in \mathbb{N}}\left\{w^{m} \in B^{L-\mathcal{J}}\left(w^{0}, w^{0}-w\right)\right\}$ and $\lim _{m \rightarrow \infty} d\left(w^{m}, w\right)=0$.

Remark 12.1 Let $U=\{0,3,6\}$. By (12.2), $7=D_{1}^{L-\mathcal{J}}(U, U) \neq 0$.

Example 12.2 Let $(X, d)$ be a complete metric space where $X=[0 ; 6]$ and $d: X^{2} \rightarrow[0 ; \infty)$ is of the form $d(x, y)=|x-y|, x, y \in X$, and let $(X, T)$ be defined by

$$
T(x)=\left\{\begin{array}{lll}
{[1 ; 2]} & \text { for } x \in(0 ; 3) \cup(3 ; 6), & \\
{[1 ; 2] \cup[4 ; 5]} & \text { for } x=3, \\
{[4 ; 5]} & \text { for } x \in\{0,6\}, &
\end{array} \quad x \in X .\right.
$$

We see that, for each $x \in X, T(x) \in C l^{L-\mathcal{P}}(X)=C l^{R-\mathcal{P}}(X)$ where $\mathcal{P}=\{d\}$. Moreover, $\operatorname{Fix}(T)=[1 ; 2]$. However, for each $\lambda \in[0 ; 1)$, condition $(1.3)$ for $(X, T)$ does not hold. We argue by contradiction and suppose that

$$
\exists_{\lambda \in[0 ; 1)} \forall_{x, y \in X}\left\{H^{d}(T(x), T(y)) \leq \lambda d(x, y)\right\} .
$$

Consider then the case when $x_{0}=2$ and $y_{0}=0$. Then we deduce the following: (i) For $u \in$ $T\left(x_{0}\right)=[1 ; 2]$, we have $d\left(u, T\left(y_{0}\right)\right)=d(u,[4 ; 5])=4-u$ and $\sup \left\{d\left(u, T\left(y_{0}\right)\right): u \in T\left(x_{0}\right)\right\}=3$; (ii) For $v \in T\left(y_{0}\right)=[4 ; 5]$, we have $d\left(v, T\left(x_{0}\right)\right)=d(v,[1 ; 2])=v-2$ and $\sup \left\{d\left(v, T\left(x_{0}\right)\right): v \in\right.$ $\left.T\left(y_{0}\right)\right\}=3$; (iii) Consequently,

$$
\begin{aligned}
& \forall \lambda \in[0 ; 1)\left\{3=H^{d}\left(T\left(x_{0}\right), T\left(y_{0}\right)\right)=\max \left\{\sup _{u \in T\left(x_{0}\right)} d\left(u, T\left(y_{0}\right)\right), \sup _{v \in T\left(y_{0}\right)} d\left(T\left(x_{0}\right), v\right)\right\}\right. \\
& \left.\quad \leq \lambda d\left(x_{0}, y_{0}\right)<d\left(x_{0}, y_{0}\right)=d(2,0)=2\right\},
\end{aligned}
$$

which is absurd. 
Remark 12.2 Observe that $(X, d)$ and $(X, T)$ defined in Examples 12.1 and 12.2 are identical. However, Example 12.1 shows that we may apply Theorem 10.1 with $\mathcal{J}$ defined by (12.2) and satisfying $\mathcal{J} \neq \mathcal{P}=\{d\}$, and Example 12.2 shows that we do not apply Theorem 1.2 of Nadler since (1.3) does not hold.

Example 12.3 Let $X=[0 ; 6]$ and let $\mathcal{P}=\{p\}$ where $p$ is a quasi-pseudometric on $X$ defined by

$$
p(x, y)= \begin{cases}0 & \text { if } x \geq y \\ 1 & \text { if } x<y\end{cases}
$$

Let $E=[0 ; 3) \cup(3 ; 6]$ and let $\mathcal{J}=\{J\}$ where $J$ is of the form

$$
J(x, y)= \begin{cases}p(x, y) & \text { if } E \cap\{x, y\}=\{x, y\} \\ 2 & \text { if } E \cap\{x, y\} \neq\{x, y\} .\end{cases}
$$

Define $(X, T)$ by

$$
T(x)= \begin{cases}{[4 ; 6]} & \text { for } x \in[0 ; 3) \cup(3 ; 6] \\ {[5 ; 6]} & \text { for } x=3\end{cases}
$$

(III.1) $\mathcal{J}$ is not symmetric. In fact, by $(12.3), J(0,4)=1$ and $J(4,0)=0$.

(III.2) $(X, \mathcal{P})$ is a quasi-gauge space and $\mathcal{J}=\{J\} \in \mathbb{J}_{(X, \mathcal{P})}^{L} \cap \mathbb{J}_{(X, \mathcal{P})}^{R}$. See Example 4.1.

(III.3) The property $T: X \rightarrow C L^{L-\mathcal{P}}(X)$ holds. This follows from (12.3) and Definitions 6.1 and $5.1(\mathrm{C})$.

(III.4) The set-valued dynamic system $(X, T)$ is a $\left(\mathcal{D}_{1}^{L-\mathcal{J}}, \lambda=1 / 2\right)$-contraction on $X$, i.e., $\forall_{x, y \in X}\left\{D_{1}^{L-\mathcal{J}}(T(x), T(y)) \leq(1 / 2) J(x, y)\right\}$, where

$$
D_{1}^{L-\mathcal{J}}(U, V)=\max \left\{\sup _{u \in U} J(u, V), \sup _{v \in V} J(U, v)\right\}, \quad U, V \in 2^{X} .
$$

Indeed, denoting $D_{1}^{L-\mathcal{J}}=D_{1}$, we see that this follows from (III.1)-(III.3) and from Cases III.4.1-III.4.3 below.

Case III.4.1. If $x, y \in[0 ; 3) \cup(3 ; 6]$, then $x, y \in E, T(x)=T(y)=[4 ; 6]=U \subset E$ and, by (12.3), $\forall_{u \in U}\{\inf \{J(u, v): v \in U\}=J(u, u)=p(u, u)=0\}$. Thus $D_{1}(T(x), T(y))=0 \leq \lambda J(x, y)$.

Case III.4.2. If $x \in[0 ; 3) \cup(3 ; 6]$ and $y=3$, then $x \in E, y \notin E, J(x, y)=2, T(x)=[4 ; 6]=$ $U \subset E$ and $T(y)=[5 ; 6]=V \subset E$. Hence, by (12.3), $u \in U$ implies

$$
\inf \{J(u, v)=p(u, v): v \in V\}= \begin{cases}1 & \text { whenever } u \in[4 ; 5) \\ 0 & \text { whenever } u \in[5 ; 6]\end{cases}
$$

On the other hand, $v \in V$ implies $\inf \{J(u, v)=p(u, v): u \in U\}=0$. Therefore, $D_{1}(T(x)$, $T(y))=1=\lambda J(x, y)$.

Case III.4.3. If $x=3$ and $y \in[0 ; 3) \cup(3 ; 6]$, then $x \notin E, y \in E, J(x, y)=2, T(x)=[5 ; 6]=U \subset$ $E$ and $T(y)=[4 ; 6]=V \subset E$. Consequently, by (12.3), $u \in U$ implies $\inf \{(u, v)=p(u, v): v \in$ $V\}=0$. Next, by (12.3), $v \in V$ implies $\inf \{J(u, v): u \in U\} \leq 1$. Therefore, $D_{1}(T(x), T(y))=$ $1=\lambda J(x, y)$. 
Case III.4.4. If $x=y=3$, then $J(x, y)=2, T(x)=T(y)=[5 ; 6]=U \subset E$ and $\forall_{u \in U}\{\inf \{J(u$, $v)=p(u, v): v \in U\}=p(u, u)=0\}$. Thus, $D_{1}(T(x), T(y))=0<\lambda J(x, y)$.

(III.5) Property (10.1) holds, i.e., $\forall_{x \in X} \forall_{\gamma \in(0 ; \infty)} \exists_{y \in T(x)}\{J(x, y)<J(x, T(x))+\gamma\}$. Indeed, this follows from Cases III.5.1-III.5.3 below.

Case III.5.1. If $x_{0} \in[0 ; 3) \cup(3 ; 4)$ and $y_{0}=4 \in T\left(x_{0}\right)=[4 ; 6]$, then $J\left(x_{0}, y_{0}\right)=p\left(x_{0}, 4\right)=1$, $J\left(x_{0}, T\left(x_{0}\right)\right)=1$ and $\forall_{\gamma \in(0 ; \infty)}\left\{J\left(x_{0}, y_{0}\right)<J\left(x_{0}, T\left(x_{0}\right)\right)+\gamma\right\}$.

Case III.5.2. If $x_{0} \in[4 ; 6]$ and $y_{0}=4 \in T\left(x_{0}\right)=[4 ; 6]$, then $J\left(x_{0}, y_{0}\right)=p\left(x_{0}, y_{0}\right)=0$, $J\left(x_{0}, T\left(x_{0}\right)\right)=0$ and $\forall_{\gamma \in(0 ; \infty)}\left\{J\left(x_{0}, y_{0}\right)<J\left(x_{0}, T\left(x_{0}\right)\right)+\gamma\right\}$.

Case III.5.3. If $x_{0}=3$ and $y_{0} \in T\left(x_{0}\right)=[5 ; 6]$, then $J\left(x_{0}, y_{0}\right)=J\left(x_{0}, T\left(x_{0}\right)\right)=2$ and $\forall_{\gamma \in(0 ; \infty)}\left\{J\left(x_{0}, y_{0}\right)<J\left(x_{0}, T\left(x_{0}\right)\right)+\gamma\right\}$.

(III.6) The set-valued dynamic system $(X, T)$ is left $\mathcal{J}$-admissible in $X$. We verify that if $w^{0} \in X$ and $\left(w^{m}: m \in\{0\} \cup \mathbb{N}\right)$ are arbitrary and fixed and such that

$$
\forall_{m \in\{0\} \cup \mathbb{N}}\left\{w^{m+1} \in T\left(w^{m}\right)\right\}
$$

and

$$
\lim _{m \rightarrow \infty} \sup _{n>m} J\left(w^{m}, w^{n}\right)=0,
$$

then

$$
\lim _{m \rightarrow \infty} J\left(w, w^{m}\right)=0 \quad \text { where } w=6
$$

In fact, first note that

$$
T^{[m]}(X)=[4 ; 6] \subset E \quad \text { for } m \geq 2 \text {. }
$$

Next we see that (12.7) is equivalent to $\forall_{\varepsilon>0} \exists_{m_{0} \in \mathbb{N}} \forall_{n>m \geq m_{0}}\left\{J\left(w^{m}, w^{n}\right)<\varepsilon\right\}$ and so, in particular in view of (12.9), (12.3) and (12.4), this implies

$$
\forall_{0<\varepsilon<1} \exists_{m_{1} \geq m_{0}} \forall_{n>m \geq m_{1}}\left\{J\left(w^{m}, w^{n}\right)=p\left(w^{m}, w^{n}\right)=0<\varepsilon\right\} .
$$

Now in view of (12.9), (12.10), (12.3) and (12.4), we conclude that $\forall_{m \geq m_{1}}\left\{w^{m} \geq w^{m+1}\right\}$ and hence, since $\forall_{m}\left\{6 \geq w^{m}\right\}$ and $6 \in E$, we must have $\lim _{m \rightarrow \infty} p\left(w, w^{m}\right)=0$ where $w=6$, and this implies (12.8). Therefore $(X, T)$ is left $\mathcal{J}$-admissible in $X$.

(III.7) The set-valued dynamic system $(X, T)$ is a left $\mathcal{P}$-quasi-closed map in $X$. Indeed, let $\left(x_{m}: m \in \mathbb{N}\right) \subset T(X)=[4 ; 6]$ be a left $\mathcal{P}$-converging sequence in $X$. Since $[4 ; 6] \in C l^{L-\mathcal{P}}(X)$, thus $\exists_{x \in T(X)=[4 ; 6]}\left\{\lim _{m \rightarrow \infty} p\left(x, x_{m}\right)=0\right\}$. In other words, $\exists_{x \in T(X)=[4 ; 6]} \forall_{\varepsilon>0} \exists_{m_{0}} \forall_{m \geq m_{0}}\{p(x$, $\left.\left.x_{m}\right)<\varepsilon\right\}$ and thus, by (12.4) and (12.3), we obtain $\exists_{x \in T(X)=[4 ; 6]} \forall_{0<\varepsilon<1} \exists_{m_{1} \geq m_{0}} \forall_{m \geq m_{1}}\left\{p\left(x, x_{m}\right)=\right.$ $0<\varepsilon\}$ or, equivalently, $\exists_{x \in T(X)=[4 ; 6]} \exists_{m_{1}} \forall_{m \geq m_{1}}\left\{x \geq x_{m}\right\}$. Of course, then also $[x ; 6] \subset$ $S_{\left(x_{m}: m \in \mathbb{N}\right)}^{L-\mathcal{P}}$. We remark that the considerations above show that if $\left(v_{m}: m \in \mathbb{N}\right)$ and $\left(u_{m}:\right.$ $m \in \mathbb{N})$ are arbitrary and fixed subsequences of $\left(x_{m}: m \in \mathbb{N}\right)$ satisfying $\forall_{m \in \mathbb{N}}\left\{v_{m} \in T\left(u_{m}\right)\right\}$, then $\exists_{m_{1}} \forall_{m \geq m_{1}} \forall_{w \in[x ; 6]}\left\{u_{m} \in[4 ; 6] \wedge v_{m} \in T\left(u_{m}\right) \wedge w \geq u_{m} \wedge w \geq v_{m} \wedge w \in T(w)\right\}$.

(III.8) For $\mathcal{J}=\{J\}$ defined by (12.3) and (12.4), all the assumptions of Theorem 10.1 in the case of left are satisfied. This follows from (III.1)-(III.7). 
We conclude that $\operatorname{Fix}(T)=[4 ; 6]$, and we claim that if $w^{0} \in X, w^{1} \in T\left(w^{0}\right), w^{2} \in T\left(w^{1}\right)$ and $x \in[4 ; 6]$ are arbitrary and fixed, and $\forall_{m \geq 3}\left\{w^{m}=x\right\}$, then the sequence $\left(w^{m}: m \in\right.$ $\{0\} \cup \mathbb{N}$ ) is a dynamic process of $T$ starting at $w^{0}$ and left $\mathcal{P}$-converging to each point $w \in[x ; 6]$ and these points $w$ satisfy $w \in T(w)$.

Remark 12.3 Let a quasi-gauge space $(X, \mathcal{P})$ and $\mathcal{J}=\{J\}$-family be as in Example 12.3.

(a) From Cases III.4.2 and III.4.3 it follows that $1=D_{1}^{L-\mathcal{J}}(U, V) \neq D_{1}^{L-\mathcal{J}}(V, U)=0$ for $U=[4 ; 6]$ and $V=[5 ; 6]$.

(b) Observe that $2=D_{1}^{L-\mathcal{J}}(U, U) \neq 0$ if $U=\{3\}$.

Example 12.4 Let a quasi-pseudometric space $(X, \mathcal{P}), \mathcal{P}=\{p\}$, and a set-valued dynamic system $(X, T)$ be as in Example 12.3. Observe that $\exists_{\lambda \in[0 ; 1)} \forall_{x, y \in X}\left\{D_{1}^{L-\mathcal{P}}(T(x), T(y)) \leq\right.$ $\lambda p(x, y)\}$ does not hold; here $D_{1}^{L-\mathcal{P}}(U, V)=\max \left\{\sup _{u \in U} p(u, V), \sup _{v \in V} p(U, v)\right\}, U, V \in 2^{X}$. In fact, we argue by contradiction and suppose that the above condition holds. Then we remark that for $x_{0}=4$ and $y_{0}=3$, we have $p\left(x_{0}, y_{0}\right)=0, T\left(x_{0}\right)=[4 ; 6]$ and $T\left(y_{0}\right)=$ [5;6]. Thus, for $u=4 \in T\left(x_{0}\right)$, we get $\inf \left\{p(4, v): v \in T\left(y_{0}\right)\right\}=1$. This shows that $1=$ $D_{1}^{L-\mathcal{P}}\left(T\left(x_{0}\right), T\left(y_{0}\right)\right) \leq \lambda p\left(x_{0}, y_{0}\right)=0$, which is absurd.

Remark 12.4 Observe that $(X, p)$ and $(X, T)$ defined in Examples 12.3 and 12.4 are identical, note that we may apply Theorem 10.1 with $\mathcal{J}$ defined by (12.3) and (12.4) and satisfying $\mathcal{J} \neq \mathcal{P}=\{p\}$ and note, however, that we do not apply Theorem 10.1 with $\mathcal{J}=\mathcal{P}=\{p\}$. Thus the existence of a $\mathcal{J}$-family such that $\mathcal{J} \neq \mathcal{P}$ is essential.

Example 12.5 Let $X=[0 ; 1]$ and let $(X, d)$ be a metric space where $d: X^{2} \rightarrow[0 ; \infty)$ is of the form

$$
d(x, y)=|x-y|, \quad x, y \in X
$$

Defining the set

$$
A=\left\{1 / 2^{n}: n \in \mathbb{N}\right\},
$$

we consider two maps $T_{i}: X \rightarrow 2^{X}, i \in\{1,2\}$, defined by:

$$
\begin{aligned}
& T_{1}(x)=(0 ; 1) \backslash A \quad \text { for } x \in X, \\
& T_{2}(x)=A \quad \text { for } x \in X .
\end{aligned}
$$

Observe that

$$
\forall_{i \in\{1,2\}} \forall_{\lambda \in[0 ; 1)} \forall_{x, y \in X}\left\{H^{d}\left(T_{i}(x), T_{i}(y)\right)=0 \leq \lambda d(x, y)\right\} .
$$

Remark 12.5 We claim that for $(X, d)$ and for $\left(X, T_{i}\right), i \in\{1,2\}$, defined in Example 12.5, we do not use Theorem 1.2 of Nadler. Indeed, we note that $(X, d)$ is complete, $(12.15)$ holds, $\operatorname{Fix}\left(T_{1}\right)=(0 ; 1) \backslash A$ and $\operatorname{Fix}\left(T_{2}\right)=A$, whereas, for each $x \in X$ and for each $i \in\{1,2\}, T_{i}(x)$ is not closed in $(X, d)$. 
Example 12.6 Let $X=[0 ; 1]$. Define $p: X^{2} \rightarrow[0 ; \infty)$ by

$$
p(x, y)= \begin{cases}0 & \text { if } x=y \text { or } A \cap\{x, y\}=\{x, y\} \\ 1 & \text { if } x \neq y \text { and } A \cap\{x, y\} \neq\{x, y\}\end{cases}
$$

here $A$ is defined by (12.12). Let $\left(X, T_{i}\right), i=1,2$, be as in (12.13) and (12.14).

(VI.1) The map $p$ defined by (12.16) is quasi-pseudometric on $X$ and $(X, \mathcal{P}), \mathcal{P}=\{p\}$, is a quasi-gauge space. See [57, (VIII.1), p.23].

(VI.2) The space $(X, \mathcal{P})$ is a left $\mathcal{P}$-sequentially complete. See [57, (IX.2), p.24].

(VI.3) For $i \in\{1,2\},\left(X, T_{i}\right)$ has the property $T_{i}: X \rightarrow C l^{L-\mathcal{P}}(X)=C l^{R-\mathcal{P}}(X)$. Indeed, let $i \in\{1,2\}$, let $x \in X$ be an arbitrary and fixed point of $X$, and let $\left(x_{m}: m \in \mathbb{N}\right)$ be an arbitrary and fixed sequence in the set $T_{i}(x)$ which is left $\mathcal{P}$-convergent to each point of a nonempty set $S_{\left(x_{m}: m \in \mathbb{N}\right)}^{L-\mathcal{P}} \subset X$. Thus we see that $\lim _{m \rightarrow \infty} p\left(w, x_{m}\right)=0$ if $w \in S_{\left(x_{m}: m \in \mathbb{N}\right)}^{L-\mathcal{P}}$ and, by (12.16), we conclude that $\forall_{0<\varepsilon<1} \exists_{m_{0}} \forall_{m \geq m_{0}}\left\{p\left(w, x_{m}\right)=0<\varepsilon\right\}$.

As a consequence we have the following cases.

Case VI.3.1. If $i=1$, then $\left(x_{m}: m \in \mathbb{N}\right) \subset T_{1}(x)=(0 ; 1) \backslash A$ and, by (12.16), $\exists_{m_{1}} \forall_{m \geq m_{1}}\left\{x_{m}=\right.$ $w\}$ and $S_{\left(x_{m}: m \in \mathbb{N}\right)}^{L-\mathcal{P}}=\{w\} \subset(0 ; 1) \backslash A=T_{1}(x)$;

Case VI.3.2. If $i=2$, then $\left(x_{m}: m \in \mathbb{N}\right) \subset T_{2}(x)=A$ and, by (12.16), $S_{\left(x_{m}: m \in \mathbb{N}\right)}^{L-\mathcal{P}}=A=T_{2}(x)$; i.e., $\forall_{w \in A}\left\{\lim _{m \rightarrow \infty} p\left(w, x_{m}\right)=0\right\}$.

(VI.4) For $i=1,2$, the set-valued dynamic system $\left(X, T_{i}\right)$ is left partially $\mathcal{P}$-admissible in $X$. In fact, observing that $T_{1}^{[m]}(X)=(0 ; 1) \backslash A$ and $T_{2}^{[m]}(X)=A$ for $m \in \mathbb{N}$, it remains to verify that if $w^{0} \in X$ and $\left(w^{m}: m \in\{0\} \cup \mathbb{N}\right)$ are such that

$$
\forall_{m \in\{0\} \cup \mathbb{N}}\left\{w^{m+1} \in T_{1}\left(w^{m}\right) \subset(0 ; 1) \backslash A\right\}
$$

or

$$
\forall_{m \in\{0\} \cup \mathbb{N}}\left\{w^{m+1} \in T_{2}\left(w^{m}\right) \subset A\right\}
$$

and

$$
\lim _{m \rightarrow \infty} \sup _{n>m} p\left(w^{m}, w^{n}\right)=0,
$$

then

$$
\exists_{w \in X}\left\{\lim _{m \rightarrow \infty} p\left(w, w^{m}\right)=\lim _{m \rightarrow \infty} p\left(w^{m}, w\right)=0\right\}
$$

here we remark that by (12.16) property (12.19) shows that

$$
\forall_{0<\varepsilon<1} \exists_{m_{1} \geq m_{0}} \forall_{n>m \geq m_{1}}\left\{p\left(w^{m}, w^{n}\right)=0<\varepsilon\right\} .
$$

One way to check this is as follows: If $i=1$, then by (12.16), (12.17) and (12.21), $\exists_{w \in(0 ; 1) \backslash A} \exists_{m_{1} \in \mathbb{N}} \forall_{m \geq m_{1}}\left\{w^{m}=w\right\}$ and $\lim _{m \rightarrow \infty} p\left(w, w^{m}\right)=0$. If $i=2$, then by (12.16), (12.18) and (12.21), we have $\forall_{w \in A}\left\{\lim _{m \rightarrow \infty} p\left(w, w^{m}\right)=0\right\}$. By symmetry of $p$, this shows that (12.20) holds. 
(VI.5) Let $i \in\{1,2\}$. For each $\lambda \in[0 ; 1)$, the set-valued dynamic system $\left(X, T_{i}\right)$ is a $\left(\mathcal{D}_{1}^{L-\mathcal{P}}, \lambda\right)$-contraction on $X$. Indeed, we see that, for each $x, y \in X$,

$$
\begin{aligned}
& \forall_{u \in T_{1}(x)=(0 ; 1) \backslash A}\left\{\inf \left\{p(u, v): v \in T_{1}(y)=(0 ; 1) \backslash A\right\}=p(u, u)=0\right\} \\
& \forall_{u \in T_{2}(x)=A}\left\{\inf \left\{p(u, v): v \in T_{2}(y)=A\right\}=p(u, v)=0\right\} .
\end{aligned}
$$

Therefore, $\forall_{i \in\{1,2\}} \forall_{\lambda \in[0 ; 1)} \forall_{x, y \in X}\left\{D_{1}^{L-\mathcal{P}}\left(T_{i}(x), T_{i}(y)\right)=0 \leq \lambda p(x, y)\right\}$.

(VI.6) For $i \in\{1,2\}$, the set-valued dynamic system $\left(X, T_{i}\right)$ is left $\mathcal{P}$-quasi-closed on $X$. Indeed, let $i \in\{1,2\}$. Let $\left(x_{m}: m \in \mathbb{N}\right)$ be an arbitrary and fixed sequence in $T_{i}(X)$, left $\mathcal{P}$-convergent to each point of a nonempty set $S_{\left(x_{m}: m \in \mathbb{N}\right)}^{L \mathcal{P}} \subset X$ and having subsequences $\left(v_{m}: m \in \mathbb{N}\right)$ and $\left(u_{m}: m \in \mathbb{N}\right)$ satisfying $\forall_{m \in \mathbb{N}}\left\{v_{m} \in T_{i}\left(u_{m}\right)\right\}$. Of course, $S_{\left(x_{m}: m \in \mathbb{N}\right)}^{L-\mathcal{P}} \subset$ $S_{\left(v_{m}: m \in \mathbb{N}\right)}^{L-\mathcal{P}}$ and $S_{\left(x_{m}: m \in \mathbb{N}\right)}^{L-\mathcal{P}} \subset S_{\left(u_{m}: m \in \mathbb{N}\right)}^{L-\mathcal{P}}$. Let now $w \in S_{\left(x_{m}: m \in \mathbb{N}\right)}^{L-\mathcal{P}}$ be arbitrary and fixed. Since $\lim _{m \rightarrow \infty} p\left(w, x_{m}\right)=\lim _{m \rightarrow \infty} p\left(w, v_{m}\right)=\lim _{m \rightarrow \infty} p\left(w, u_{m}\right)=0$, thus, by (12.16),

$$
\forall_{0<\varepsilon<1} \exists_{m_{0}} \forall_{m \geq m_{0}}\left\{p\left(w, x_{m}\right)=0<\varepsilon \wedge p\left(w, v_{m}\right)=0<\varepsilon \wedge p\left(w, u_{m}\right)=0<\varepsilon\right\} .
$$

As a consequence, we have the following cases.

Case VI.6.1. If $i=1$, then by (12.13), (12.16) and (12.21), $\exists_{m_{0}} \forall_{m \geq m_{0}}\left\{x_{m}=v_{m}=u_{m}=w\right\}$. Hence, $w \in(0 ; 1) \backslash A, S_{\left(x_{m}: m \in \mathbb{N}\right)}^{L-\mathcal{P}}=S_{\left(v_{m}: m \in \mathbb{N}\right)}^{L-\mathcal{P}}=S_{\left(u_{m}: m \in \mathbb{N}\right)}^{L-\mathcal{P}}=\{w\}$ and $w \in T(w)$. This gives, by Section 8 , that $T_{1}$ is left $\mathcal{P}$-quasi-closed on $X$.

Case VI.6.2. If $i=2$, then by (12.14), (12.16) and (12.21), $\exists_{m_{0}} \forall_{m \geq m_{0}}\left\{x_{m}, v_{m}, u_{m} \in A\right\}$. Hence, $w \in A=S_{\left(x_{m}: m \in \mathbb{N}\right)}^{L-\mathcal{P}}=S_{\left(v_{m}: m \in \mathbb{N}\right)}^{L \mathcal{P}}=S_{\left(u_{m}: m \in \mathbb{N}\right)}^{L-\mathcal{P}}$ and $w \in T_{2}(w)$. This gives, by Section 8, that $T_{2}$ is left $\mathcal{P}$-quasi-closed on $X$.

(VI.7) For each $i \in\{1,2\}$, property (10.1) holds, i.e.,

$$
\forall_{x \in X} \forall_{\gamma \in(0 ; \infty)} \exists_{y \in T_{i}(x)}\left\{p(x, y)<p\left(x, T_{i}(x)\right)+\gamma\right\} .
$$

Indeed, this follows from Cases VI.7.1 and VI.7.2 below.

Case VI.7.1. Let $x_{0} \in A$.

If $y_{0}=2 / 3 \in T_{1}\left(x_{0}\right)=(0 ; 1) \backslash A$, then $p\left(x_{0}, y_{0}\right)=1, p\left(x_{0}, T\left(x_{0}\right)\right)=1$ and $\forall_{\gamma \in(0 ; \infty)}\left\{p\left(x_{0}, y_{0}\right)<\right.$ $\left.p\left(x_{0}, T_{1}\left(x_{0}\right)\right)+\gamma\right\}$.

If $y_{0} \in T_{2}\left(x_{0}\right)=A$, then $p\left(x_{0}, y_{0}\right)=0, p\left(x_{0}, T_{2}\left(x_{0}\right)\right)=0$ and $\forall_{\gamma \in(0 ; \infty)}\left\{p\left(x_{0}, y_{0}\right)<p\left(x_{0}\right.\right.$, $\left.\left.T_{2}\left(x_{0}\right)\right)+\gamma\right\}$

Case VI.7.2. Let $x_{0} \in(0 ; 1) \backslash A$.

If $y_{0}=x_{0} \in T_{1}\left(x_{0}\right)=(0 ; 1) \backslash A$, then $p\left(x_{0}, y_{0}\right)=1, p\left(x_{0}, T_{1}\left(x_{0}\right)\right)=1$ and $\forall_{\gamma \in(0 ; \infty)}\left\{p\left(x_{0}, y_{0}\right)<\right.$ $\left.p\left(x_{0}, T_{1}\left(x_{0}\right)\right)+\gamma\right\}$.

If $y_{0} \in T_{2}\left(x_{0}\right)=A$, then $p\left(x_{0}, y_{0}\right)=1, p\left(x_{0}, T_{2}\left(x_{0}\right)\right)=1$ and $\forall \gamma \in(0 ; \infty)\left\{p\left(x_{0}, y_{0}\right)<p\left(x_{0}\right.\right.$, $\left.\left.T_{2}\left(x_{0}\right)\right)+\gamma\right\}$.

(VI.8) All the assumptions of Theorem 10.1(B) when $s=1$ are satisfied. This is proved in (VI.1)-(VI.7).

We conclude that $\operatorname{Fix}\left(T_{1}\right)=(0 ; 1) \backslash A$ and $\operatorname{Fix}\left(T_{2}\right)=A$, and one shows the following.

Claim VI.8.1. Let $w^{0} \in X$. If $w \in(0 ; 1) \backslash A$ and $w^{m+1} \in T_{1}\left(w^{m}\right), m \in\{0\} \cup \mathbb{N}$, satisfies $\forall_{m \in \mathbb{N}}\left\{w^{m}=w\right\}$, then the sequence $\left(w^{m}: m \in\{0\} \cup \mathbb{N}\right)$ is left $\mathcal{P}$-convergent to $w$ and $w \in$ $T_{1}(w)$. Moreover, $\left(w^{m}: m \in \mathbb{N}\right) \subset B^{L-\mathcal{P}}\left(w^{0}, 1\right)$ and $w, w^{0} \in B^{L-\mathcal{P}}\left(w^{0}, 1\right)$ whenever $w^{0} \neq w$, and, for each $r \in(0 ; \infty),\left(w^{m}: m \in \mathbb{N}\right) \subset B^{L-\mathcal{P}}\left(w^{0}, r\right)$ and $w, w^{0} \in B^{L-\mathcal{P}}\left(w^{0}, r\right)$ whenever $w^{0}=w$; 
Claim VI.8.2. Let $w^{0} \in X$. If $w \in A$ and $w^{m+1} \in T_{2}\left(w^{m}\right), m \in\{0\} \cup \mathbb{N}$, satisfies $\forall_{m \in \mathbb{N}}\left\{w^{m} \in\right.$ $A\}$, then the sequence $\left(w^{m}: m \in\{0\} \cup \mathbb{N}\right)$ is left $\mathcal{P}$-convergent to $w$ and $w \in T_{2}(w)$. Moreover, $\left(w^{m}: m \in \mathbb{N}\right) \subset B^{L-\mathcal{P}}\left(w^{0}, 1\right)$ and $w, w^{0} \in B^{L-\mathcal{P}}\left(w^{0}, 1\right)$ whenever $w^{0} \in X \backslash A$, and, for each $r \in(0 ; \infty),\left(w^{m}: m \in \mathbb{N}\right) \subset B^{L-\mathcal{P}}\left(w^{0}, r\right)$ and $w, w^{0} \in B^{L-\mathcal{P}}\left(w^{0}, r\right)$ whenever $w^{0} \in A$.

Remark 12.6 Let $X=[0 ; 1]$, let $\left(X, T_{i}\right), i \in\{1,2\}$, be defined by (12.13) and (12.14), let $d$ be of the form (12.11) and let $p$ be of the form (12.16). We point out some facts concerning Examples 12.5 and 12.6 .

(a) $(X, \mathcal{P})$ is not Hausdorff. Indeed, if $x, y \in A$ and $x \neq y$, then $p(x, y)=p(y, x)=0$, and we notice that evidently (2.1) does not hold.

(b) The basic idea of Example 12.6 is as follows. First, in Example 12.5, in the metric space $(X, d)$, we showed that it is not possible to use the metric structure on $X$ determined by $d$ despite the fact that (12.15) and thus also (1.3) holds for $\left(X, T_{i}\right)$, $i \in\{1,2\}$. Next, by a suitable choice of the not Hausdorff structure on $X$, defined by $\mathcal{P}=\{p\}$, we proved that for $(X, \mathcal{P})$ and $\left(X, T_{i}\right), i \in\{1,2\}$, the assumptions of Theorem 10.1(B) when $s=1$ hold and we can then apply this theorem.

Example 12.7 Let $X=[0 ; 1]$ and let $A$ and $p: X^{2} \rightarrow[0 ; \infty)$ be defined by $(12.12)$ and (12.16), respectively. For $W \subset A, W \neq \varnothing, W \neq A$, we define the map

$$
T(x)= \begin{cases}W & \text { for } x \in X \backslash W \\ A \backslash W & \text { for } x \in W\end{cases}
$$

(VII.1) We claim that, for each $\lambda \in[0 ; 1),(X, T)$ is a $\left(\mathcal{D}_{1}^{L-\mathcal{P}}, \lambda\right)$-contraction and $\left(\mathcal{D}_{1}^{R-\mathcal{P}}, \lambda\right)$ contraction on $X$. In view of (12.16), this follows from the fact that $\forall_{\lambda \in[0 ; 1)} \forall_{x, y \in X}\left\{D_{1}^{L-\mathcal{P}}(T(x)\right.$, $\left.T(y))=D_{1}^{R-\mathcal{P}}(T(x), T(y)) \leq \lambda p(x, y)\right\}$. Indeed, by (12.23), $x \in X$ implies $T(x) \subset A$. Hence, by (12.16) we have that

$$
\begin{aligned}
& \forall_{x, y \in X}\left\{D_{1}^{L-\mathcal{P}}(T(x), T(y))=D_{1}^{R-\mathcal{P}}(T(x), T(y))\right. \\
& \left.\quad=\max \left\{\sup _{u \in T(x) \subset A} p(u, T(y)), \sup _{v \in T(y) \subset A} p(T(x), v)\right\}=0\right\} .
\end{aligned}
$$

(VII.2) The set-valued dynamic system $(X, T)$ is left and right partially $\mathcal{P}$-admissible in $X$. In fact, since $T^{[m]}(X)=A$ for $m \in \mathbb{N}$, thus if $w^{0} \in X$ and $\left(w^{m}: m \in\{0\} \cup \mathbb{N}\right)$ are arbitrary and fixed and such that $\forall_{m \in\{0\} \cup \mathbb{N}}\left\{w^{m+1} \in T\left(w^{m}\right)\right\}$, then $\forall_{m \in\{0\} \cup \mathbb{N}}\left\{w^{m+1} \in A\right\}$ and $\lim _{m \rightarrow \infty} \sup _{n>m} p\left(w^{m}, w^{n}\right)=0$ (thus also $\lim _{m \rightarrow \infty} \sup _{n>m} p\left(w^{n}, w^{m}\right)=0$ ). Consequently, by (12.16), $\forall_{w \in A}\left\{\lim _{m \rightarrow \infty} p\left(w, w^{m}\right)=\lim _{m \rightarrow \infty} p\left(w^{m}, w\right)=0\right\}$. By Definition 7.1(B), $(X, T)$ is left and right partially $\mathcal{P}$-admissible in $X$.

(VII.3) The set-valued dynamic system $\left(X, T^{[2]}\right)$,

$$
T^{[2]}(x)= \begin{cases}A \backslash W & \text { for } x \in X \backslash W, \\ W & \text { for } x \in W\end{cases}
$$

is left and right $\mathcal{P}$-quasi-closed on $X$. Indeed, let $\left(x_{m}: m \in \mathbb{N}\right)$ be an arbitrary and fixed sequence in $T^{[2]}(X)=A$, left $\mathcal{P}$-convergent to each point $w$ of a nonempty set $S_{\left(x_{m}: m \in \mathbb{N}\right)}^{L-\mathcal{P}}$ and having subsequences $\left(v_{m}: m \in \mathbb{N}\right)$ and $\left(u_{m}: m \in \mathbb{N}\right)$ satisfying $\forall_{m \in \mathbb{N}}\left\{v_{m} \in T^{[2]}\left(u_{m}\right)\right\}$. 
It follows from (12.16) that then $w \in S_{\left(x_{m}: m \in \mathbb{N}\right)}^{L-\mathcal{P}}=S_{\left(v_{m}: m \in \mathbb{N}\right)}^{L-\mathcal{P}}=S_{\left(u_{m}: m \in \mathbb{N}\right)}^{L-\mathcal{P}}=A$ and $w \in T^{[2]}(w)$. Analogously, we prove that $\left(X, T^{[2]}\right)$ is right $\mathcal{P}$-quasi-closed on $X$.

(VII.4) Properties (10.1) and (10.2) hold. Indeed, this follows from Cases VII.4.1-VII.4.3 below.

Case VII.4.1. If $x_{0} \in A$ and $y_{0} \in T\left(x_{0}\right)$, then $T\left(x_{0}\right) \subset A, p\left(x_{0}, y_{0}\right)=0, p\left(x_{0}, T\left(x_{0}\right)\right)=0$ and $\forall_{\gamma \in(0 ; \infty)}\left\{p\left(x_{0}, y_{0}\right)<p\left(x_{0}, T\left(x_{0}\right)\right)+\gamma\right\}$

Case VII.4.2. If $x_{0} \in X \backslash A$ and $y_{0} \in T\left(x_{0}\right)$, then $T\left(x_{0}\right) \subset A, p\left(x_{0}, y_{0}\right)=1, p\left(x_{0}, T\left(x_{0}\right)\right)=1$ and $\forall_{\gamma \in(0 ; \infty)}\left\{p\left(x_{0}, y_{0}\right)<p\left(x_{0}, T\left(x_{0}\right)\right)+\gamma\right\}$.

Case VII.4.3. Finally, $p\left(x_{0}, y_{0}\right)=p\left(y_{0}, x_{0}\right)$ and $p\left(x_{0}, T\left(x_{0}\right)\right)=p\left(T\left(x_{0}\right), x_{0}\right)$ follow from the fact that $\mathcal{P}$ is symmetric.

(VII.5) All the assumptions of Theorem 10.1(B) when $s=2$ are satisfied. This is proved in (VII.1)-(VII.4).

We conclude that $\operatorname{Fix}(T)=\varnothing$ and $\operatorname{Fix}\left(T^{[2]}\right)=A$, and one shows the following.

Claim VII.5.1. If $w^{0} \in A, w \in \operatorname{Fix}\left(T^{[2]}\right)$ and $w^{m+1} \in T\left(w^{m}\right), m \in\{0\} \cup \mathbb{N}$, then $w \in A$, $\forall_{m \in \mathbb{N}}\left\{w^{m} \subset A\right\}$, the sequence $\left(w^{m}: m \in\{0\} \cup \mathbb{N}\right)$ is left and right $\mathcal{P}$-convergent to $w$, and, for each $r \in(0 ; \infty),\left(w^{m}: m \in \mathbb{N}\right) \subset B$ and $w, w^{0} \in B$; here $B=B^{L-\mathcal{P}}\left(w^{0}, r\right)=B^{R-\mathcal{P}}\left(w^{0}, r\right)$

Claim VII.5.2. If $w^{0} \in X \backslash A, w \in \operatorname{Fix}\left(T^{[2]}\right)$ and $w^{m+1} \in T^{[2]}\left(w^{m}\right), m \in\{0\} \cup \mathbb{N}$, then $w \in A$, $\forall_{m \in \mathbb{N}}\left\{w^{m} \in A\right\}$, the sequence $\left(w^{m}: m \in\{0\} \cup \mathbb{N}\right)$ is left $\mathcal{P}$-convergent to $w,\left(w^{m}: m \in \mathbb{N}\right) \subset B$ and $w, w^{0} \in B$; here $B=B^{L-\mathcal{P}}\left(w^{0}, 1\right)=B^{R-\mathcal{P}}\left(w^{0}, 1\right)$.

Remark 12.7 We point out some facts concerning Example 12.7.

(a) $(X, \mathcal{P})$ is not Hausdorff; see Remark 12.6(a).

(b) Noting that $\mathcal{P}$ is symmetric, we see that $C l^{L-\mathcal{P}}(X)=C l^{R-\mathcal{P}}(X)$ holds.

(c) The property $T: X \rightarrow C l^{L-\mathcal{P}}(X)$ does not hold. In fact, it is not hard to see that, for $x \in X \backslash W, T(x)=W \neq c l_{X}^{L-\mathcal{J}}(T(x))=A$ and for $x \in X \backslash W$, $T(x)=A \backslash W \neq c l_{X}^{L-\mathcal{J}}(T(x))=A$.

(d) $\operatorname{Fix}(T)=\varnothing$ and $\operatorname{Fix}\left(T^{[2]}\right) \neq \varnothing$.

\section{Competing interests}

The author declares that he has no competing interests.

Received: 17 July 2014 Accepted: 16 November 2014 Published: 05 Dec 2014

\section{References}

1. Banach, S: Sur les opérations dans les ensembles abstraits et leurs applications aux équations intégrales. Fundam. Math. 3, 133-181 (1922)

2. Nadler, SB: Multi-valued contraction mappings. Pac. J. Math. 30, 475-488 (1969)

3. Alemany, S, Romaguera, AS: On right K-sequentially complete quasi-metric spaces. Acta Math. Hung. 75, 267-278 (1997)

4. Aubin, JP, Frankowska, H: Set-Valued Analysis. Birkhäuser, Boston (1990)

5. Aubin, JP, Siegel, J: Fixed points and stationary points of dissipative multivalued maps. Proc. Am. Math. Soc. 78, 391-398 (1980)

6. Aydi, H, Abbas, M, Vetro, C: Partial Hausdorff metric and Nadler's fixed point theorem on partial metric spaces. Topol. Appl. 159, 3234-3242 (2012)

7. Benavides, TD, Ramírez, PL: Fixed point theorems for multivalued nonexpansive mappings satisfying inwardness conditions. J. Math. Anal. Appl. 291, 100-108 (2004)

8. de Blasi, FS, Myjak, J, Reich, S, Zaslawski, AJ: Generic existence and approximation of fixed points for nonexpansive set-valued maps. Set-Valued Var. Anal. 17, 97-112 (2009)

9. Caristi, J: Fixed point theorems for mappings satisfying inwardness conditions. Trans. Am. Math. Soc. 215, 241-251 (1976)

10. Caristi, J, Kirk, WA: Geometric Fixed Point Theory and Inwardness Conditions. Lecture Notes in Math., vol. 490, pp. 74-83. Springer, Berlin (1975)

11. Ćirić, L: Multi-valued nonlinear contraction mappings. Nonlinear Anal. 71, 2716-2723 (2009)

12. Covitz, H, Nadler, SB: Multi-valued contraction mappings in generalized metric spaces. Isr. J. Math. 8, 5-11 (1970) 
13. Ekeland, I: Remarques sur les problémes variationnels. I. C. R. Acad. Sci. Paris Sér. A-B 275, 1057-1059 (1972)

14. Ekeland, I: On the variational principle. J. Math. Anal. Appl. 47, 324-353 (1974)

15. Ekeland, I: Nonconvex minimization problems. Bull. Am. Math. Soc. 1, 443-474 (1979)

16. Espínola, R, Petruşel, A: Existence and data dependence of fixed points for multivalued operators on gauge spaces. J. Math. Anal. Appl. 309, 420-432 (2005)

17. Espínola, R, Kirk, WA: Set-valued contractions and fixed points. Nonlinear Anal. 54, 485-494 (2003)

18. Feng, Y, Liu, S: Fixed point theorems for multi-valued contractive mappings and multi-valued Caristi type mappings. J. Math. Anal. Appl. 317, 103-112 (2006)

19. Frigon, M: Fixed point results for multivalued maps in metric spaces with generalized inwardness conditions. Fixed Point Theory Appl. 2010, Article ID 183217 (2010)

20. Frigon, M: Fixed point results for generalized contractions in gauge spaces with applications. Proc. Am. Math. Soc $128,2957-2965$ (2000)

21. Frigon, M: Fixed point results for multivalued contractions on gauge spaces. In: Set-Valued Mappings with Applications in Nonlinear Analysis. Ser. Math. Anal. Appl., vol. 4, pp. 175-181. Taylor \& Francis, London (2002)

22. Frigon, M: Fixed point and continuation results for contractions in metric and gauge spaces. Banach Cent. Publ. 77, 89-114 (2007)

23. Kada, O, Suzuki, T, Takahashi, W: Nonconvex minimization theorems and fixed point theorems in complete metric spaces. Math. Jpn. 44, 381-391 (1996)

24. Kirk, WA: Fixed points of asymptotic contractions. J. Math. Anal. Appl. 277, 645-650 (2003)

25. Kikkawa, M, Suzuki, T: Three fixed point theorems for generalized contractions with constants in complete metric spaces. Nonlinear Anal. 69, 2942-2949 (2008)

26. Leader, S, Hoyle, SL: Contractive fixed points. Fundam. Math. 87, 93-108 (1975)

27. Lin, L-J, Du, W-S: Ekeland's variational principle, minimax theorems and existence of nonconvex equilibria in complete metric spaces. J. Math. Anal. Appl. 323, 360-370 (2006)

28. Mizoguchi, N, Takahashi, W: Fixed point theorems for multivalued mappings on complete metric spaces. J. Math. Anal. Appl. 141, 177-188 (1989)

29. Nadler, SB: Periodic points of multi-valued $\varepsilon$-contractive maps. Topol. Methods Nonlinear Anal. 22, 399-409 (2003)

30. Pathak, HK, Shahzad, N: Fixed point results for set-valued contractions by altering distances in complete metric spaces. Nonlinear Anal. 70, 2634-2641 (2009)

31. Petruşel, G: Fixed point results for multivalued contractions on ordered gauge spaces. Cent. Eur. J. Math. 7, 520-528 (2009)

32. Quantina, K, Kamran, T: Nadler's type principle with hight order of convergence. Nonlinear Anal. 69, 4106-4120 (2008)

33. Reich, S: Fixed points of contractive functions. Boll. Unione Mat. Ital. 5, 26-42 (1972)

34. Reilly, IL: A generalized contraction principle. Bull. Aust. Math. Soc. 10, 349-363 (1974)

35. Reilly, IL, Subrahmanyam, PV, Vamanamurthy, MK: Cauchy sequences in quasi-pseudo-metric spaces. Monatshefte Math. 93, 127-140 (1982)

36. Smithson, RE: Fixed points for contractive multifunctions. Proc. Am. Math. Soc. 27, 192-194 (1971)

37. Suzuki, T: Generalized distance and existence theorems in complete metric spaces. J. Math. Anal. Appl. 253, 440-458 (2001)

38. Suzuki, T: Several fixed point theorems concerning $\tau$-distance. Fixed Point Theory Appl. 2004, 195-209 (2004)

39. Suzuki, T: Several fixed point theorems in complete metric spaces. Yokohama Math. J. 44, 61-72 (1997)

40. Suzuki, T: Mizoguchi-Takahashi's fixed point theorem is a real generalization of Nadler's. J. Math. Anal. Appl. 340 752-755 (2008)

41. Suzuki, T: Kannan mappings are contractive mappings in some sense. Comment. Math. 45, 43-56 (2005)

42. Tarafdar, E, Yuan, GX-Z: Set-valued contraction mapping principle. Appl. Math. Lett. 8, 79-81 (1995)

43. Takahashi, W: Existence theorems generalizing fixed point theorems for multivalued mappings. In: Baillon, JB, Théra, M (eds.) Fixed Point Theory and Applications (Marseille, 1989). Pitman Res. Notes Math. Ser., vol. 252, pp. 397-406. Longman Sci. Tech., Harlow (1991)

44. Tarafdar, E: An approach to fixed-point theorems on uniform space. Trans. Am. Math. Soc. 191, $209-225$ (1974)

45. Tataru, D: Viscosity solutions of Hamilton-Jacobi equations with unbounded nonlinear terms. J. Math. Anal. Appl. 163 345-392 (1992)

46. Vályi, I: A general maximality principle and a fixed point theorem in uniform spaces. Period. Math. Hung. 16, 127-134 (1985)

47. Włodarczyk, K, Plebaniak, R: Maximality principle and general results of Ekeland and Caristi types without lower semicontinuity assumptions in cone uniform spaces with generalized pseudodistances. Fixed Point Theory Appl. 2010, Article ID 175453 (2010)

48. Włodarczyk, K, Plebaniak, R: Periodic point, endpoint, and convergence theorems for dissipative set-valued dynamic systems with generalized pseudodistances in cone uniform and uniform spaces. Fixed Point Theory Appl. 2010, Article ID 864536 (2010)

49. Włodarczyk, K, Plebaniak, R, Obczyński, C: Convergence theorems, best approximation and best proximity for set-valued dynamic systems of relatively quasi-asymptotic contractions in cone uniform spaces. Nonlinear Anal. 72 , 794-805 (2010)

50. Włodarczyk, K, Plebaniak, R: Quasi-gauge spaces with generalized quasi-pseudodistances and periodic points of dissipative set-valued dynamic systems. Fixed Point Theory Appl. 2011, Article ID 712706 (2011)

51. Włodarczyk, K, Plebaniak, R: Contractivity of Leader type and fixed points in uniform spaces with generalized pseudodistances. J. Math. Anal. Appl. 387, 533-541 (2012)

52. Włodarczyk, K, Plebaniak, R: Generalized uniform spaces, uniformly locally contractive set-valued dynamic systems and fixed points. Fixed Point Theory Appl. 2012, Article ID 104 (2012)

53. Włodarczyk, K, Plebaniak, R: Leader type contractions, periodic and fixed points and new completivity in quasi-gauge spaces with generalized quasi-pseudodistances. Topol. Appl. 159, 3504-3512 (2012)

54. Włodarczyk, K, Plebaniak, R: Fixed points and endpoints of contractive set-valued maps in cone uniform spaces with generalized pseudodistances. Fixed Point Theory Appl. 2012, Article ID 176 (2012) 
55. Włodarczyk, K, Plebaniak, R: Asymmetric structures, discontinuous contractions and iterative approximation of fixed and periodic points. Fixed Point Theory Appl. 2013, Article ID 128 (2013)

56. Włodarczyk, K, Plebaniak, R: Contractions of Banach, Tarafdar, Meir-Keeler, Ćirić-Jachymski-Matkowski and Suzuki types and fixed points in uniform spaces with generalized pseudodistances. J. Math. Anal. Appl. 404, 338-350 (2013)

57. Włodarczyk, K, Plebaniak, R: New completeness and periodic points of discontinuous contractions of Banach-type in quasi-gauge spaces without Hausdorff property. Fixed Point Theory Appl. 2013, Article ID 289 (2013)

58. Yuan, GX-Z: KKM Theory and Applications in Nonlinear Analysis. Dekker, New York (1999)

59. Zhong, C-H, Zhu, J, Zhao, P-H: An extension of multi-valued contraction mappings and fixed points. Proc. Am. Math. Soc. 128, 2439-2444 (1999)

60. Aubin, JP, Ekeland, J: Applied Nonlinear Analysis. Wiley, New York (1984)

61. Dugundji, J: Topology. Allyn \& Bacon, Boston (1966)

62. Reilly, IL: Quasi-gauge spaces. J. Lond. Math. Soc. 6, 481-487 (1973)

10.1186/1687-1812-2014-239

Cite this article as: Włodarczyk: Hausdorff quasi-distances, periodic and fixed points for Nadler type set-valued contractions in quasi-gauge spaces. Fixed Point Theory and Applications 2014, 2014:239

\section{Submit your manuscript to a SpringerOpen ${ }^{\ominus}$ journal and benefit from:}

- Convenient online submission

- Rigorous peer review

- Immediate publication on acceptance

- Open access: articles freely available online

- High visibility within the field

- Retaining the copyright to your article 